\title{
AN ORDER-REVERSING DUALITY MAP FOR CONJUGACY CLASSES IN LUSZTIG'S CANONICAL QUOTIENT
}

\author{
PRAMOD N. ACHAR
}

\begin{abstract}
We define a partial order on the set $\mathcal{N}_{\mathrm{o}, \overline{\mathrm{c}}}$ of pairs $(\mathcal{O}, C)$, where $\mathcal{O}$ is a nilpotent orbit and $C$ is a conjugacy class in $\bar{A}(\mathcal{O})$, Lusztig's canonical quotient of $A(\mathcal{O})$. We then show that there is a unique order-reversing duality map $\mathcal{N}_{\mathrm{o}, \overline{\mathrm{c}}} \rightarrow{ }^{L} \mathcal{N}_{\mathrm{O}, \overline{\mathrm{c}}}$ that has certain properties analogous to those of the original Lusztig-Spaltenstein duality map. This generalizes work of E. Sommers.
\end{abstract}

\section{INTRODUCTION}

Let $G$ be a connected simple complex algebraic group, and let $\mathfrak{g}$ be its Lie algebra. Let $\mathcal{N}$ be the nilpotent cone in $\mathfrak{g}$; let $\mathcal{N}_{\mathrm{o}}$ be the set of $G$-orbits in $\mathcal{N}$. The notion of a duality map for nilpotent orbits has its roots in the introduction of "special representations" of a Weyl group by Lusztig [11]. He gave a purely algebraic treatment aimed at studying primitive ideals in enveloping algebras, but in passing, he conjectured that (and it was quickly verified that) all special representations should be assigned to nilpotent orbits with the trivial local system via Springer's correspondence, so that special representations would sit in bijection with a remarkable set of "special" nilpotent orbits, denoted $\mathcal{N}_{\mathrm{o}}^{\mathrm{sp}}$. Subsequently, Lusztig and Spaltenstein observed that the set of special nilpotent orbits admits a natural order-reversing bijection (with respect to the usual closure order on nilpotent orbits) that usually corresponds, in the language of special representations, to tensoring with the sign representation. (There are a couple of curious exceptions to this in types $E_{7}$ and $E_{8}$.) Indeed, this bijection could be extended to an order-reversing map $d_{\mathrm{LS}}: \mathcal{N}_{\mathrm{o}} \rightarrow \mathcal{N}_{\mathrm{o}}$ whose image consists precisely of the special orbits, and which is an involution when restricted to its image. In [20], Spaltenstein gives an axiomatic treatment of the map $d_{\mathrm{LS}}$, showing that it is the unique map satisfying certain order conditions and a certain compatibility with induction.

Now, since a group $G$ and its Langlands dual ${ }^{L} G$ have isomorphic Weyl groups, there is a natural bijection between their respective sets of special nilpotent orbits $\mathcal{N}_{\mathrm{o}}^{\mathrm{sp}}$ and ${ }^{{ }} \mathcal{N}_{\mathrm{o}}^{\mathrm{sp}}$. Spaltenstein observed that this bijection is order-preserving, so by composing $d_{\mathrm{LS}}$ with it, one obtains a map $\mathcal{N}_{\mathrm{O}} \rightarrow{ }^{L} \mathcal{N}_{\mathrm{o}}$ or ${ }^{L} \mathcal{N}_{\mathrm{o}} \rightarrow \mathcal{N}_{\mathrm{o}}$. Barbasch and Vogan later gave an elegant and intrinsic construction of this incarnation of the map, which we shall denote by $d_{\mathrm{BV}}$, in terms of associated varieties of certain Harish-Chandra modules.

Sommers [18] has shown how to enlarge the domain of $d_{\mathrm{BV}}$ so that the extended map surjects onto ${ }^{L} \mathcal{N}_{\mathrm{o}}$. This latter set does not, in general, sit in bijection with $\mathcal{N}_{\mathrm{o}}$, so there is no analogue of Sommers' map for $d_{\mathrm{LS}}$. Let $A(\mathcal{O})$ be the component group of the centralizer in $G$ of some element of $\mathcal{O}$, and let $\mathcal{N}_{\mathrm{o}, \mathrm{c}}$ be the set of pairs $(\mathcal{O}, C)$, where $\mathcal{O} \in \mathcal{N}_{\mathrm{o}}$ and $C$ is a conjugacy class in $A(\mathcal{O})$. (We do not need to be careful about which element of $\mathcal{O}$ we pick to define $A(\mathcal{O})$, since any two yield component groups that are canonically isomorphic up to inner automorphism.) Sommers' map $d_{\mathrm{S}}: \mathcal{N}_{\mathrm{o}, \mathrm{c}} \rightarrow{ }^{L} \mathcal{N}_{\mathrm{o}}$ agrees with $d_{\mathrm{BV}}$ when composed with the inclusion $\mathcal{N}_{\mathrm{o}} \hookrightarrow \mathcal{N}_{\mathrm{o}, \mathrm{c}}$ defined by $\mathcal{O} \mapsto(\mathcal{O}, 1)$.

Finally, let $\bar{A}(\mathcal{O})$ be Lusztig's canonical quotient of $A(\mathcal{O})$. This was originally introduced by Lusztig [12] for special orbits, but Sommers [18], in the course of giving a new characterization of the canonical quotient, observes that the definition makes sense for all orbits. We let $\mathcal{N}_{\mathrm{o}, \overline{\mathrm{c}}}$ be the set of pairs $(\mathcal{O}, C)$, where this time $C$ is a conjugacy class in $\bar{A}(\mathcal{O})$. Sommers' description of $\bar{A}(\mathcal{O})$ leads to a proof of the following statement ([18], Proposition 15): if $C$ and $C^{\prime}$ are two conjugacy classes in $A(\mathcal{O})$ that descend to the same conjugacy class in $\bar{A}(\mathcal{O})$, then $d_{\mathrm{S}}(\mathcal{O}, C)=d_{\mathrm{S}}\left(\mathcal{O}, C^{\prime}\right)$. In other words, $d_{\mathrm{S}}$ factors through the natural projection $\mathcal{N}_{\mathrm{o}, \mathrm{c}} \rightarrow \mathcal{N}_{\mathrm{o}, \overline{\mathrm{c}}}$. In this article, we often regard $d_{\mathrm{S}}$ as a map $\mathcal{N}_{\mathrm{o}, \overline{\mathrm{c}}} \rightarrow{ }^{L} \mathcal{N}_{\mathrm{O}}$.

Date: 16 September 2002. 
One task we accomplish in this paper is the introduction of a partial order on the set $\mathcal{N}_{\mathrm{o}, \overline{\mathrm{c}}}$, as follows. We say that $(\mathcal{O}, C) \leq\left(\mathcal{O}^{\prime}, C^{\prime}\right)$ if

$$
\mathcal{O} \leq \mathcal{O}^{\prime} \quad \text { and } \quad d_{\mathrm{S}}(\mathcal{O}, C) \geq d_{\mathrm{S}}\left(\mathcal{O}^{\prime}, C^{\prime}\right)
$$

A priori, this partial order might not be well-defined: we might have had $d_{\mathrm{S}}(\mathcal{O}, C)=d_{\mathrm{S}}\left(\mathcal{O}, C^{\prime}\right)$ even when $C \neq C^{\prime}$. In the course of this paper, we rectify this by proving a converse to Proposition 15 of [18].

Theorem 1. Let $C, C^{\prime} \subset A(\mathcal{O})$ be two conjugacy classes associated to the same orbit. Then $d_{\mathrm{S}}(\mathcal{O}, C)=$ $d_{\mathrm{S}}\left(\mathcal{O}, C^{\prime}\right)$ if and only if $C$ and $C^{\prime}$ have the same image in $\bar{A}(\mathcal{O})$. As a consequence, the partial order (1) on $\mathcal{N}_{\mathrm{o}, \overline{\mathrm{c}}}$ is well-defined.

The principal aim of this paper is to show that $\mathcal{N}_{\mathrm{o}, \overline{\mathrm{c}}}$ admits a unique duality map $\bar{d}$ that is compatible with the aforementioned maps in the appropriate senses. In particular, such a duality map ought to satisfy the partial-order properties of $d_{\mathrm{LS}}$ and $d_{\mathrm{BV}}$ :

(1) If $(\mathcal{O}, C) \leq\left(\mathcal{O}^{\prime}, C^{\prime}\right)$, then $\bar{d}(\mathcal{O}, C) \geq \bar{d}\left(\mathcal{O}^{\prime}, C^{\prime}\right)$.

(2) $\bar{d}^{2}(\mathcal{O}, C) \geq(\mathcal{O}, C)$.

It also ought to coincide with $d_{\mathrm{BV}}$ and $d_{\mathrm{S}}$ when its domain or codomain is restricted. Indeed, we need only make an explicit requirement with respect to $d_{\mathrm{S}}$; that automatically implies the desired compatibility with $d_{\mathrm{BV}}$ as well. We write $p r_{1}: \mathcal{N}_{\mathrm{o}, \overline{\mathrm{c}}} \rightarrow \mathcal{N}_{\mathrm{o}}$ for the obvious projection.

(3) $p r_{1} \circ \bar{d}(\mathcal{O}, C)=d_{\mathrm{S}}(\mathcal{O}, C)$.

Finally, we need one additional condition to guarantee the uniqueness of the map.

(4) Among maps respecting the first three axioms, $\bar{d}$ has an image set of maximal size.

To be precise, we ought to be seeking a pair of maps $\bar{d}: \mathcal{N}_{\mathrm{o}, \overline{\mathrm{c}}} \rightarrow{ }^{L} \mathcal{N}_{\mathrm{o}, \overline{\mathrm{c}}}, \bar{d}:{ }^{L} \mathcal{N}_{\mathrm{o}, \overline{\mathrm{c}}} \rightarrow \mathcal{N}_{\mathrm{o}, \overline{\mathrm{c}}}$, both of which satisfy the above axioms. Indeed, axiom (2) only makes sense if we have two such maps together. Nevertheless, to avoid making the language too cumbersome, we will speak throughout the paper of "a" duality map $\mathcal{N}_{\mathrm{o}, \overline{\mathrm{c}}} \rightarrow{ }^{L} \mathcal{N}_{\mathrm{o}, \overline{\mathrm{c}}}$, and always assume it to be implicitly accompanied by a partner map ${ }^{L} \mathcal{N}_{\mathrm{o}, \overline{\mathrm{c}}} \rightarrow \mathcal{N}_{\mathrm{o}, \overline{\mathrm{c}}}$.

The main result of the paper is the following.

Theorem 2. There is a unique map $\bar{d}: \mathcal{N}_{\mathrm{o}, \overline{\mathrm{c}}} \rightarrow{ }^{L} \mathcal{N}_{\mathrm{o}, \overline{\mathrm{c}}}$ satisfying the axioms (1)-(4).

Let us call this map the extended duality map.

In type $A$, of course, all the $\bar{A}(\mathcal{O})$-groups are trivial, so this theorem does not say anything new: the extended duality map is just the same as $d_{\mathrm{BV}}$. In all other types, the theorem will be proved by giving an explicit construction of the map. For the classical types, this entails a combinatorial algorithm in terms of partitions, whereas in the exceptional groups, we define $\bar{d}$ simply by tabulating all its values.

We begin our discussion in Section 2 by collecting some properties that must be satisfied by any putative extended duality map. These lead up to a criterion for showing that a candidate map satisfies axiom (4), and that it is the unique such map. In Section 3, we define the combinatorial objects that will be used to work with $\mathcal{N}_{\mathrm{O}}$ and $\mathcal{N}_{\mathrm{o}, \overline{\mathrm{c}}}$ in the classical groups, and we recall various useful facts about them. Serious work on the classical-groups case begins in Section 4, where we give the definitions of $\bar{d}$ and develop some basic techniques for studying it. Section 5 contains the proof of Theorems 1 and 2 for the classical groups. In Section 6 , we consider the exceptional groups, for which the main theorems are proved simply by drawing out the partial-order diagrams and verifying the existence of the extended duality map by inspection. Additionally, these partial-order diagrams are accompanied by those for a few classical groups of low rank, simply for the sake of having some examples to look at. Finally, in Section 7, we explore some possible applications and consequences of the present work.

I would like to thank A.-M. Aubert, R. Bezrukavnikov, V. Ginzburg, R. Kottwitz, V. Ostrik, and D. Vogan for helpful conversations. I would like to specifically thank one of my referees for proposing axiom (4): an earlier draft of this work employed a different statement, which did not imply uniqueness. Finally, I would especially like to thank E. Sommers. His paper [18] provides the bulk of the motivation for this one; this paper would not have been possible in the absence of the numerous discussions I have had with him on these topics. 


\section{Formal properties of DUALity}

Throughout this section, we assume that Theorem 1 is true, so the partial order on $\mathcal{N}_{\mathrm{o}, \overline{\mathrm{c}}}$ is defined, and the axioms for an extended duality map make sense. We begin a few easy properties of this partial order.

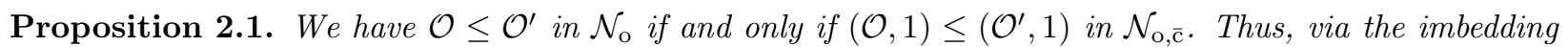
$\mathcal{O} \mapsto(\mathcal{O}, 1)$, the partially ordered set $\mathcal{N}_{\mathrm{O}}$ can be regarded as a subset of $\mathcal{N}_{\mathrm{O}, \overline{\mathrm{c}}}$ with the inherited partial order.

Proof. From the definition of the partial order, we know that $(\mathcal{O}, 1) \leq\left(\mathcal{O}^{\prime}, 1\right)$ implies that $\mathcal{O} \leq \mathcal{O}^{\prime}$. For the converse, we need to prove that if $\mathcal{O} \leq \mathcal{O}^{\prime}$, then $d_{\mathrm{S}}(\mathcal{O}, 1) \geq d_{\mathrm{S}}\left(\mathcal{O}^{\prime}, 1\right)$. But we know that $d_{\mathrm{S}}(\mathcal{O}, 1)=d_{\mathrm{BV}}(\mathcal{O})$ and $d_{\mathrm{S}}\left(\mathcal{O}^{\prime}, 1\right)=d_{\mathrm{BV}}\left(\mathcal{O}^{\prime}\right)$, and we further know that $\mathcal{O} \leq \mathcal{O}^{\prime}$ implies $d_{\mathrm{BV}}(\mathcal{O}) \geq d_{\mathrm{BV}}\left(\mathcal{O}^{\prime}\right)$.

Proposition 2.2. Regarding the Sommers duality map $d_{\mathrm{S}}$ as being a map $\mathcal{N}_{\mathrm{O}, \overline{\mathrm{c}}} \rightarrow{ }^{L} \mathcal{N}_{\mathrm{O}}$, we have that $(\mathcal{O}, C) \leq\left(\mathcal{O}^{\prime}, C^{\prime}\right)$ implies $d_{\mathrm{S}}(\mathcal{O}, C) \geq d_{\mathrm{S}}\left(\mathcal{O}^{\prime}, C^{\prime}\right)$. That is, $d_{\mathrm{S}}$ is an order-reversing map.

Proof. This is an obvious consequence of the definition of the partial order on $\mathcal{N}_{\mathrm{o}, \overline{\mathrm{c}}}$.

Proposition 2.3. For a fixed orbit $\mathcal{O}$ and any conjugacy class $C \subset \bar{A}(\mathcal{O})$, we have $(\mathcal{O}, 1) \leq(\mathcal{O}, C)$.

Proof. (This fact is hinted at in [18], where it is proved that $(\mathcal{O}, 1)$ has minimal $\tilde{b}$-value among all the $(\mathcal{O}, C)$.) All we have to check is that $d_{\mathrm{S}}(\mathcal{O}, 1) \geq d_{\mathrm{S}}(\mathcal{O}, C)$. In the exceptional groups, we can verify this simply by scanning Sommers' tables of computed values from [18]. In the classical groups, it is an easy computation from Sommers' formulas for $d_{\mathrm{S}}$, which we recall at the end of Section 3. We defer carrying out the computation until then.

We now turn our attention to duality maps. Some formal properties can be deduced from just the first three axioms. Let us define a weak extended duality map to be any map $\bar{d}: \mathcal{N}_{\mathrm{O}, \overline{\mathrm{c}}} \rightarrow{ }^{L} \mathcal{N}_{\mathrm{O}, \overline{\mathrm{c}}}$ satisfying the first three axioms, but not necessarily the fourth. Let us say that a pair $(\mathcal{O}, C)$ is special for $\bar{d}$, or simply special if no ambiguity is likely, if it is in the image of $\bar{d}$.

Proposition 2.4. We have $\bar{d}^{3}=\bar{d}$, so that when we restrict to the special set, the map $\bar{d}$ is an orderreversing bijection between special pairs in $\mathcal{N}_{\mathrm{o}, \overline{\mathrm{c}}}$ and those in ${ }^{L} \mathcal{N}_{\mathrm{o}, \overline{\mathrm{c}}}$, and $\bar{d}^{2}$ is the identity map. In general, $\bar{d}^{2}(\mathcal{O}, C)$ is the unique smallest special pair that is greater than or equal to $(\mathcal{O}, C)$.

Proof. Axiom (2) says that $\bar{d}^{2}(\mathcal{O}, C) \geq(\mathcal{O}, C)$. Applying $\bar{d}$ to both sides of this, we obtain $\bar{d}^{3}(\mathcal{O}, C) \leq$ $\bar{d}(\mathcal{O}, C)$, by axiom (1). But on the other hand, axiom $(2)$ also tells us that $\bar{d}^{2}(\bar{d}(\mathcal{O}, C)) \geq \bar{d}(\mathcal{O}, C)$. We conclude that $\bar{d}^{3}(\mathcal{O}, C)=\bar{d}(\mathcal{O}, C)$.

For the second part of the proposition, we know that $\bar{d}^{2}(\mathcal{O}, C)$ is special and greater than or equal to $(\mathcal{O}, C)$. Now, let $\left(\mathcal{O}^{\prime}, C^{\prime}\right) \geq(\mathcal{O}, C)$ be any special pair. We have $\bar{d}\left(\mathcal{O}^{\prime}, C^{\prime}\right) \leq \bar{d}(\mathcal{O}, C)$, whence $\bar{d}^{2}\left(\mathcal{O}^{\prime}, C^{\prime}\right) \geq \bar{d}^{2}(\mathcal{O}, C)$. But since $\left(\mathcal{O}^{\prime}, C^{\prime}\right)$ is special, we have $\bar{d}^{2}\left(\mathcal{O}^{\prime}, C^{\prime}\right)=\left(\mathcal{O}^{\prime}, C^{\prime}\right)$, so we can deduce that $\left(\mathcal{O}^{\prime}, C^{\prime}\right) \geq \bar{d}^{2}(\mathcal{O}, C)$ : thus $\bar{d}^{2}(\mathcal{O}, C)$ is the smallest special pair that is greater than or equal to $(\mathcal{O}, C)$.

Proposition 2.5. If $\bar{d}_{1}, \bar{d}_{2}: \mathcal{N}_{\mathrm{O}, \overline{\mathrm{c}}} \rightarrow{ }^{L} \mathcal{N}_{\mathrm{O}, \overline{\mathrm{c}}}$ are two weak extended duality maps giving rise to the same special set, then $\bar{d}_{1}=\bar{d}_{2}$.

Proof. We first show that $\bar{d}_{1}$ and $\bar{d}_{2}$ agree on special pairs. Suppose $(\mathcal{O}, C)$ is special, and that $\mathcal{O}^{\prime}=$ $d_{\mathrm{S}}(\mathcal{O}, C)$. We must have $\bar{d}_{1}(\mathcal{O}, C)=\left(\mathcal{O}^{\prime}, C_{1}\right), \bar{d}_{2}(\mathcal{O}, C)=\left(\mathcal{O}^{\prime}, C_{2}\right)$ for some $C_{1}$ and $C_{2}$. Moreover, the preceding proposition tells us that $\bar{d}_{1}\left(\mathcal{O}^{\prime}, C_{1}\right)=(\mathcal{O}, C)=\bar{d}_{2}\left(\mathcal{O}^{\prime}, C_{2}\right)$. Applying axiom $(3)$ again, we have $d_{\mathrm{S}}\left(\mathcal{O}^{\prime}, C_{1}\right)=d_{\mathrm{S}}\left(\mathcal{O}^{\prime}, C_{2}\right)=\mathcal{O}$. Finally, Theorem 1 says that we must have $C_{1}=C_{2}$.

Second, if $(\mathcal{O}, C)$ is nonspecial, Proposition 2.4 tells us that there exists a unique smallest special pair $\left(\mathcal{O}_{0}, C_{0}\right)$ that is larger than $(\mathcal{O}, C)$, and that $\bar{d}_{1}(\mathcal{O}, C)=\bar{d}_{1}\left(\mathcal{O}_{0}, C_{0}\right)=\bar{d}_{2}\left(\mathcal{O}_{0}, C_{0}\right)=\bar{d}_{2}(\mathcal{O}, C)$.

Within the proof of this last proposition lurks an important observation: all weak extended duality maps $\bar{d}$ for which a given pair $(\mathcal{O}, C)$ is special take the same value on it. This is because, by Theorem 1 , there is at most one class $C^{\prime}$ such that $d_{\mathrm{S}}\left(\mathcal{O}^{\prime}, C^{\prime}\right)=\mathcal{O}$, where $\mathcal{O}^{\prime}=d_{\mathrm{S}}(\mathcal{O}, C)$. If there does not exist such a $C^{\prime}$, then $(\mathcal{O}, C)$ cannot be special for any weak extended duality map. Inspired by this, we define the set

$$
\mathcal{N}_{\mathrm{o}, \overline{\mathrm{c}}}^{\mathrm{sp}}=\left\{(\mathcal{O}, C) \in \mathcal{N}_{\mathrm{o}, \overline{\mathrm{c}}} \mid \text { there exists a } C^{\prime} \text { such that } d_{\mathrm{S}}\left(\mathcal{O}^{\prime}, C^{\prime}\right)=\mathcal{O} \text {, where } \mathcal{O}^{\prime}=d_{\mathrm{S}}(\mathcal{O}, C)\right\},
$$

and note that the special set of any weak extended duality map must be contained within $\mathcal{N}_{\mathrm{o}, \overline{\mathrm{c}}}^{\mathrm{sp}}$. If there exists one whose special set is the entirety of $\mathcal{N}_{\mathrm{o}, \overline{\mathrm{c}}}^{\mathrm{sp}}$, then it would automatically satisfy the fourth axiom as 
well. It would also be the unique possible extended duality map, by Proposition 2.5. We have established the following.

Proposition 2.6. If $\bar{d}$ is a weak extended duality map whose special set is $\mathcal{N}_{\mathrm{o}, \overline{\mathrm{c}}}^{\mathrm{sp}}$, then $\bar{d}$ is in fact the unique extended duality map.

The proof of Theorem 2 in Sections 5 and 6 is carried out by explicitly constructing a weak extended duality map $\bar{d}$ which happens to have all of $\mathcal{N}_{\mathrm{o}, \overline{\mathrm{c}}}^{\mathrm{sp}}$ as its special set, and then applying the preceding proposition. Although that construction is a laborious undertaking which occupies most of this paper, there is a posteriori a concise, uniform description of the extended duality map. It is the map whose image is ${ }^{L} \mathcal{N}_{\mathrm{o}, \overline{\mathrm{c}}}^{\mathrm{sp}}$, and whose values are computed according to the discussion in the proof of Proposition 2.5.

We conclude this section with a few additional observations about the extended duality map. Below, $\bar{d}$ will denote only the extended duality map, and "special" will refer to all elements of $\mathcal{N}_{\mathrm{o}, \overline{\mathrm{c}}}^{\mathrm{sp}}$.

Proposition 2.7. Any pair of the form $(\mathcal{O}, 1)$ is special.

Proof. Let $\mathcal{O}^{\prime}=d_{\mathrm{S}}(\mathcal{O}, 1)=d_{\mathrm{BV}}(\mathcal{O})$. To show that $(\mathcal{O}, 1) \in \mathcal{N}_{\mathrm{o}, \overline{\mathrm{c}}}^{\mathrm{sp}}$, we must merely demonstrate the existence of a class $C^{\prime}$ such that $d_{\mathrm{S}}\left(\mathcal{O}^{\prime}, C^{\prime}\right)=\mathcal{O}$. This was done by Sommers in [18] with his construction of a "canonical inverse": this is a certain right inverse to $d_{\mathrm{S}}$ that was used to show that $d_{\mathrm{S}}$ is surjective. The details of the construction are such that the preimage produced for $\mathcal{O}$ is always a conjugacy class associated to $d_{\mathrm{BV}}(\mathcal{O})$.

The following two statements are easily deduced from the above uniform description of $\bar{d}$.

Proposition 2.8. Sommers' canonical inverse is given by $\mathcal{O} \mapsto \bar{d}(\mathcal{O}, 1)$.

Proposition 2.9. An orbit $\mathcal{O}$ is special if and only if $\bar{d}(\mathcal{O}, 1)=\left(d_{\mathrm{BV}}(\mathcal{O}), 1\right)$.

Even if $\mathcal{O}$ is a special orbit, we cannot say anything in general about whether $(\mathcal{O}, C)$ is a special pair for nontrivial $C$. The computed examples in Section 6 include instances of both special and nonspecial pairs of this form.

\section{Orbits, PARTITIONS, AND COMPONENT GROUPS}

We spend this section collecting facts and formulas for working with partitions as a way of understanding nilpotent orbits in the classical groups. It is suggested that the reader skip this section, referring back to it only when necessary to find a particular definition or formula.

3.1. Partitions. Let $\mathcal{P}(n)$ be the set of partitions of $n$. For a partition $\lambda$, let $|\lambda|$ denote the sum of the parts of $\lambda$. We typically write $\lambda=\left[\lambda_{1} \geq \lambda_{2} \geq \cdots \geq \lambda_{k}\right]$, and we assume $\lambda_{k} \neq 0$ unless stated otherwise. Sometimes, however, we shall write partitions as follows, using exponents to indicate multiplicities: $\left[a_{1}^{p_{1}}, \ldots, a_{k}^{p_{k}}\right]$, with $a_{1}>\cdots>a_{k}$. Let $r_{\lambda}(a)$, or simply $r(a)$, denote the multiplicity of $a$ as a part in $\lambda$. We define the height of a part in a partition to be the number of parts greater than or equal to the given one: $\operatorname{ht}_{\lambda}(a)=\operatorname{ht}(a)=\sum_{b \geq a} r_{\lambda}(b)$. Note that this formula makes sense even if $a$ is not a part of $\lambda$; i.e., if $r_{\lambda}(a)=0$. We shall employ the notion of height in such circumstances from time to time; we may refer to it as "generalized height" to draw attention to the fact that $r_{\lambda}(a)=0$. Finally, we write $\# \lambda$ to denote the total number of parts of $\lambda$.

For odd $n$, we write $\mathcal{P}_{B}(n)$ for the set of partitions in which even parts occur with even multiplicity. For even $n$, we write $\mathcal{P}_{C}(n)$ for the set of partitions in which odd parts occur with even multiplicity, and $\mathcal{P}_{D}(n)$ for the set of partitions in which even parts occur with even multiplicity. Here, the subscript letters correspond to the type of classical Lie group whose nilpotent orbits are indexed by the given set of partitions, with one caveat: very even partitions (those consisting only of even parts with even multiplicity) in type $D$ correspond to two nilpotent orbits. We ignore this fact throughout the paper, because such orbits have trivial $A(\mathcal{O})$-groups, so the duality map we construct here will not have anything new to say about them. We will sometimes write $\mathcal{P}_{1}(n)$ for $\mathcal{P}_{C}(n)$, and $\mathcal{P}_{0}(n)$ for either $\mathcal{P}_{B}(n)$ or $\mathcal{P}_{D}(n)$. This will allow us to make concise statements about $\mathcal{P}_{\epsilon}(n)$ for $\epsilon \in\{0,1\}$.

If $\lambda=\left[\lambda_{1} \geq \cdots \geq \lambda_{k}\right]$, we write $\sigma_{j}(\lambda)$ for the $i$-th partial sum $\sum_{i=1}^{j} \lambda_{i}$. Recall the standard partial order on partitions: for $\lambda, \lambda^{\prime} \in \mathcal{P}(n)$, we say that $\lambda \leq \lambda^{\prime}$ if we have $\sigma_{j}(\lambda) \leq \sigma_{j}\left(\lambda^{\prime}\right)$ for all $j$. In this case, we say that $\lambda^{\prime}$ dominates $\lambda$. Recall also that the closure order on nilpotent orbits coincides with this order on 
partitions in the classical groups. For a partition $\lambda$, let $\lambda^{*}$ denote its transpose partition, and let $\lambda_{B}, \lambda_{C}$, $\lambda_{D}$ denote its $B$-, $C$-, and $D$-collapses respectively, whenever those are defined. (The $X$-collapse of $\lambda$ is the unique largest partition $\lambda^{\prime}$ such that $\lambda^{\prime} \leq \lambda$ and $\lambda^{\prime} \in \mathcal{P}_{X}(n)$; see [9].) Suppose $\lambda=\left[\lambda_{1} \geq \cdots \geq \lambda_{k}\right] \in \mathcal{P}(n)$, and assume that $\lambda_{k} \neq 0$. We define the following four operations:

$$
\begin{aligned}
\lambda^{+} & =\left[\lambda_{1}+1 \geq \lambda_{2} \geq \cdots \geq \lambda_{k}\right] & \lambda^{-} & =\left[\lambda_{1} \geq \cdots \geq \lambda_{k-1} \geq \lambda_{k}-1\right] \\
\lambda_{+} & =\left[\lambda_{1} \geq \cdots \geq \lambda_{k} \geq 1\right] & & \lambda_{-}=\lambda^{*-*}
\end{aligned}
$$

(Note that $\lambda_{+}=\lambda^{*+*}$ as well.)

Given two partitions $\lambda$ and $\mu$, we can form their union $\lambda \cup \mu$, a partition of $|\lambda|+|\mu|$, by putting $r_{\lambda \cup \mu}(a)=$ $r_{\lambda}(a)+r_{\mu}(a)$ for all $a$. We can also take their join, defined by

$$
\lambda \vee \mu=\left(\lambda^{*} \cup \mu^{*}\right)^{*} .
$$

If one thinks of partitions in terms of Young diagrams, the union corresponds to combining the rows of the two diagrams, while the join corresponds to combining their columns. Finally, if $\lambda=\left[\lambda_{1} \geq \cdots \geq \lambda_{k}\right]$, we define

$$
\chi_{j}^{+}(\lambda)=\left[\lambda_{1} \geq \cdots \geq \lambda_{j}\right] \quad \text { and } \quad \chi_{j}^{-}(\lambda)=\left[\lambda_{j+1} \geq \cdots \geq \lambda_{k}\right] .
$$

Note that $\lambda=\chi_{j}^{+}(\lambda) \cup \chi_{j}^{-}(\lambda)$ for any $j$.

Sometimes we will want to restrict the kinds of partitions that we take unions and joins of, in order to have control over what the union or join looks like. Given two partitions $\lambda$ and $\mu$, let $a$ be the smallest part of $\lambda$, and let $b$ be the largest part of $\mu$. We say that $\lambda$ is superior to $\mu$ if $a \geq b$. We say that $\lambda$ is evenly (resp. oddly) superior to $\mu$ if there is an even (resp. odd) number $m$ such that $a \geq m \geq b$.

3.2. Computing with collapses. The following observations about collapses will be relied upon heavily when we set about the work of proving the main theorems in Section 5. If $\lambda$ has $k$ parts, then any collapse $\lambda_{X}$ of it must have either $k$ or $k+1$ parts. Moreover, $B$-partitions necessarily have an odd number of parts, and $D$-partitions necessarily have an even number, so we can determine exactly how many parts $\lambda_{B}$ or $\lambda_{D}$ must have (of course, only one of those collapses is defined for any particular $\lambda$ ). Finally, $\lambda_{C}$ (when it is defined) must have the same number of parts as $\lambda$, because if it had one more, we would have introduced a new part equal to 1 , but we cannot create new odd parts when taking a $C$-collapse.

We will often encounter situations in which we have a partition written as the union or join of two others, and in which we will want to express a certain collapse of $\lambda$ in terms of collapses of the smaller partitions. The following proposition collects formulas for twelve kinds of joins, and twelve kinds of unions. This table of formulas is certainly sufficient for the calculations in this paper. The author has not bothered to determine whether any of the twenty-four could have been omitted.

Lemma 3.1. Suppose $\lambda=\lambda^{\prime} \vee \lambda^{\prime \prime}$. Let $k$ be the largest part of $\lambda^{\prime}$, and let $p=\left|\lambda^{\prime}\right|$. Suppose in addition that $\mu=\mu^{\prime} \cup \mu^{\prime \prime}$, that $\mu^{\prime}$ has $k$ parts, and that $\left|\mu^{\prime}\right|=p$. Assume that $\lambda^{\prime *}$ is superior to $\lambda^{\prime \prime *}$, and that $\mu^{\prime}$ is superior to $\mu^{\prime \prime}$. The following table expresses various collapses of $\lambda$ and $\mu$ in terms of collapses of the smaller partitions. For any formula containing $\lambda^{\prime \prime}{ }_{B}$, we must make the additional assumption that $\lambda^{\prime *}$ is oddly superior to $\lambda^{\prime \prime *}$; for any containing $\lambda^{\prime \prime}{ }_{D}$, we assume that $\lambda^{\prime *}$ is evenly superior to $\lambda^{\prime \prime *}$. Similarly, for any formula containing $\mu^{\prime-}$ and $\mu^{\prime \prime+}$, we must assume that $\mu^{\prime}$ is superior to $\mu^{\prime \prime+}$.

\begin{tabular}{ccccc} 
& \multicolumn{2}{c}{$k$ even } & \multicolumn{2}{c}{$k$ odd } \\
& $p$ even & $p$ odd & $p$ even & $p$ odd \\
$\lambda_{B}:$ & $\lambda^{\prime+}{ }_{B} \vee \lambda^{\prime \prime}{ }_{B}$ & $\lambda^{\prime}{ }_{B} \vee \lambda^{\prime \prime}{ }_{D}$ & $\lambda^{\prime}{ }_{B} \vee \lambda^{\prime \prime}{ }_{C}$ & $\lambda^{\prime}{ }_{B} \vee \lambda^{\prime \prime}{ }_{C}$ \\
$\lambda_{C}:$ & $\lambda^{\prime} \vee \lambda^{\prime \prime}{ }_{C}$ & $\lambda^{\prime+}{ }_{C} \vee \lambda^{\prime \prime}{ }_{C}$ & $\lambda^{\prime}{ }_{C} \vee \lambda^{\prime \prime}{ }_{D}$ & $\lambda^{\prime+}{ }_{C} \vee \lambda^{\prime \prime}{ }_{B}$ \\
$\lambda_{D}:$ & $\lambda^{\prime}{ }_{D} \vee \lambda^{\prime \prime}{ }_{D}$ & $\lambda^{\prime+}{ }_{D} \vee \vee \lambda^{\prime \prime}{ }_{B}$ & $\lambda^{\prime}{ }_{D} \vee \lambda^{\prime \prime}{ }_{C}$ & $\lambda^{\prime+}{ }_{D} \vee \lambda^{\prime \prime}{ }_{C}$ \\
& & & & \\
$\mu_{B}:$ & $\mu^{\prime} \cup{ }_{D} \cup \mu^{\prime \prime}{ }_{B}$ & $\mu^{\prime}{ }_{D} \cup \mu^{\prime \prime+}{ }_{B}$ & $\mu^{\prime}{ }_{B} \cup \mu^{\prime \prime}{ }_{D}$ & $\mu^{\prime}{ }_{B} \cup \mu^{\prime \prime}{ }_{D}$ \\
$\mu_{C}:$ & $\mu_{C}^{\prime} \cup \mu^{\prime \prime}{ }_{C}$ & $\mu^{\prime}{ }_{C} \cup \mu^{\prime+}{ }_{C}$ & $\mu^{\prime}{ }_{C} \cup \mu^{\prime \prime}{ }_{C}$ & $\mu^{\prime-}{ }_{C} \cup \mu^{\prime \prime+}{ }_{C}$ \\
$\mu_{D}:$ & $\mu^{\prime} \cup \mu^{\prime \prime}{ }_{D}$ & $\mu^{\prime-}{ }_{D} \cup \mu^{\prime \prime+}{ }_{D}$ & $\mu^{\prime-}{ }_{B} \cup \mu^{\prime \prime+}{ }_{B}$ & $\mu^{\prime}{ }_{B} \cup \mu^{\prime \prime}{ }_{B}$
\end{tabular}

Proof. Once one becomes accustomed to the pattern of producing these formulas, it is fairly easy to compute all of them. We will work through just one: that for $\lambda_{B}$ when $k$ is odd and $p$ is even. For $\lambda_{B}$ to be defined, $|\lambda|$ must be odd; and since $p$ is even, $\left|\lambda^{\prime \prime}\right|$ must be odd. Since $k$ is odd, the parities of parts of $\lambda^{\prime \prime}$ are opposite to those of the corresponding parts of $\lambda$, so taking a $B$-collapse of $\lambda$ should manifest itself as something like 
a $C$-collapse of $\lambda^{\prime \prime}$. Since $\left|\lambda^{\prime \prime}\right|$ is odd, if we attempt to take a $C$-collapse of it, we will be partway through a collapsing operation when we get to the end of the partition: there will be a leftover "1" to be added to some odd part, but no remaining odd parts to receive it. This "1" will "leak" onto $\lambda^{\prime}$. We can preemptively take care of this leaking 1 by looking at $\lambda^{\prime+}$ and $\lambda^{\prime \prime}-$ instead. Now, we comfortably take the $C$-collapse of $\lambda^{\prime \prime}-$, and the $B$-collapse of $\lambda^{\prime+}$. (If $\lambda^{\prime \prime}$ has $m$ parts, it may seem that we should have added the leaking 1 to the $(m+1)$-th part of $\lambda^{\prime}$, not its first part, as is done by writing $\lambda^{\prime+}$. But in $\lambda^{\prime+}$, the first part is now even, and the remaining parts up to the $m$-th one are all odd, so in taking a $B$-collapse, that " 1 " gets shoved down to at least the $(m+1)$-th row anyway.) We thus obtain that $\lambda_{B}=\lambda^{\prime+}{ }_{B} \vee \lambda^{\prime \prime-}{ }_{C}$.

The only comment we make on other cases is regarding the auxiliary superiority requirements. Terms of the form $\lambda^{\prime \prime}{ }_{B}$ or $\lambda^{\prime \prime}{ }_{D}$ may have a different number of parts from $\lambda^{\prime \prime}$, so we have to be a lot more careful in considering the interaction between $\lambda^{\prime}$ and $\lambda^{\prime \prime}$. The easiest thing to do is impose a condition that the largest part of $\lambda^{\prime}$ have high enough multiplicity that we need not worry: that is exactly what the superiority condition does for us. Similar considerations result in the corresponding requirements when we deal with $\mu^{\prime-}$ and $\mu^{\prime \prime+}$.

3.3. Marked partitions. If $X$ is one of $B, C$, or $D$, we define $\tilde{\mathcal{P}}_{X}(n)$ to be the set of pairs of partitions $(\nu, \eta)$, such that:

(a) $\nu \cup \eta \in \mathcal{P}_{X}(n)$.

(b) Every part of $\nu$ is odd (resp. even) if $X=B$ or $D$ (resp. $C$ ) and has multiplicity 1.

(c) If $X=B$ or $D, \nu$ has an even number of parts.

This notation is taken from [18], but we will typically find another notation far more convenient for our purposes. We will write elements $(\nu, \eta) \in \tilde{\mathcal{P}}_{X}(n)$ as ${ }^{\langle\nu\rangle} \lambda$, where $\lambda=\nu \cup \eta$. In this notation, we think of elements of $\tilde{\mathcal{P}}_{X}(n)$ just as partitions from $\mathcal{P}_{X}(n)$, with the additional data that certain parts (viz. those in $\nu$ ) have been "marked." Indeed, we will refer to elements of these sets as marked partitions, and we call $\nu$ the marking partition and $\lambda$ the underlying partition. Marked partitions of the form ${ }^{\langle\varnothing\rangle} \lambda$ are called trivially marked partitions. As before, we sometimes write $\tilde{\mathcal{P}}_{0}(n)$ and $\tilde{\mathcal{P}}_{1}(n)$ for these sets.

We can attempt to define the union and join operations for marked partitions, but the constructions we give now may not always yield a valid marked partition. This situation will be rectified in the following subsection, when we introduce "reduced marked partitions." For now, we define the union simply by

$$
{ }^{\left\langle\nu_{1}\right\rangle} \lambda_{1} \cup{ }^{\left\langle\nu_{2}\right\rangle} \lambda_{2}=\left\langle\left(\nu_{1} \cup \nu_{2}\right)\right\rangle\left(\lambda_{1} \cup \lambda_{2}\right) .
$$

Next, write $\lambda_{1} \vee \lambda_{2}=\left[a_{1} \geq \cdots \geq a_{k}\right]$. Suppose $\nu_{1}=\left[n_{1} \geq \cdots \geq n_{p}\right]$, and $\nu_{2}=\left[m_{1} \geq \cdots \geq m_{r}\right]$. We define

$$
{ }^{\left\langle\nu_{1}\right\rangle} \lambda_{1} \vee{ }^{\left\langle\nu_{2}\right\rangle} \lambda_{2}={ }^{\langle\omega\rangle}\left(\lambda_{1} \vee \lambda_{2}\right), \quad \text { where } \quad \omega=\left[a_{\mathrm{ht}_{\lambda_{1}}\left(n_{i}\right)} \mid i=1, \ldots, p\right] \cup\left[a_{\mathrm{ht}_{\lambda_{2}}\left(m_{i}\right)} \mid i=1, \ldots, r\right] .
$$

The idea of this definition is that we should preserve the heights of the marked parts when we take the join. Quite often, we will encounter joins of marked partitions in which the largest part of $\lambda_{2}$ has very high multiplicity, more than the total number of parts of $\lambda_{1}$. In this special circumstance, understanding the join of marked partitions is much easier: if $b$ is that largest part of $\lambda_{2}$, we obtain

$$
\omega=\left[b+n_{1} \geq \cdots \geq b+n_{p} \geq m_{1} \geq \cdots \geq m_{r}\right] .
$$

For $\lambda \in \mathcal{P}_{\epsilon}(n)$ and $\delta \in\{0,1\}$, let

$$
S_{\delta}(\lambda)=\{a \mid a \not \equiv \epsilon \quad(\bmod 2) \text { and } r(a) \equiv \delta \quad(\bmod 2)\} .
$$

We will just write $S_{\delta}$ when no confusion will result. For ${ }^{\langle\nu\rangle} \lambda \in \tilde{\mathcal{P}}_{\epsilon}(n)$, write

$$
T_{\delta}\left({ }^{\langle\nu\rangle} \lambda\right)=T_{\delta}=\nu \cap S_{\delta}(\lambda) .
$$

3.4. Parametrizing $\mathcal{N}_{\mathbf{o}, \mathbf{c}}$ and $\mathcal{N}_{\mathbf{o}, \overline{\mathbf{c}}}$. A detailed account of the following description of a parametrization of $\mathcal{N}_{\mathrm{o}, \mathrm{c}}$ and $\mathcal{N}_{\mathrm{o}, \overline{\mathrm{c}}}$ can be found in [17]. Now, $\tilde{\mathcal{P}}_{X}(n)$ is close to indexing the set $\mathcal{N}_{\mathrm{o}, \mathrm{c}}$ in type $X$. Actually, there is a surjective map

$$
\tilde{\mathcal{P}}_{X}(n) \rightarrow \mathcal{N}_{\mathrm{o}, \mathrm{c}}
$$

which is a bijection in type $B$, but is 2-to- 1 over any orbit in types $C$ and $D$ whose partition has $S_{1} \neq \varnothing$. There is, of course, a further projection

$$
\tilde{\mathcal{P}}_{X}(n) \rightarrow \mathcal{N}_{\mathrm{o}, \overline{\mathrm{c}}} .
$$


We now describe this projection in some detail. Given $\lambda$, list the elements of $S_{1}$ as $j_{l}>\cdots>j_{1}$. Assume that $l$ is even in type $C$ by taking $j_{1}=0$ if necessary ( $l$ is automatically odd in type $B$ and even in type $D$ ). Now, given ${ }^{\langle\nu\rangle} \lambda$, let $T_{0}^{(m)}=\left\{a \in T_{0}\left({ }^{\langle\nu\rangle} \lambda\right) \mid j_{m}<a<j_{m+1}\right\}$, and let $T_{1}^{(m)}=T_{1} \cap\left\{j_{m}\right\}$. Next, we define an equivalence relation $\sim$ on $\tilde{\mathcal{P}}_{\epsilon}(n)$ as follows: ${ }^{\langle\nu\rangle} \lambda \sim{ }^{\left\langle\nu^{\prime}\right\rangle} \lambda$ if

(a) $T_{0}^{(m)}\left({ }^{\langle\nu\rangle} \lambda\right)=T_{0}^{(m)}\left({ }^{\left\langle\nu^{\prime}\right\rangle} \lambda\right)$ whenever $m$ is even.

(b) $\left|T_{1}^{(m+1)}\left({ }^{\langle\nu\rangle} \lambda\right) \cup T_{0}^{(m)}\left({ }^{\langle\nu\rangle} \lambda\right) \cup T_{1}^{(m)}\left({ }^{\langle\nu\rangle} \lambda\right)\right| \equiv\left|T_{1}^{(m+1)}\left({ }^{\left\langle\nu^{\prime}\right\rangle} \lambda\right) \cup T_{0}^{(m)}\left({ }^{\left\langle\nu^{\prime}\right\rangle} \lambda\right) \cup T_{1}^{(m)}\left({ }^{\left\langle\nu^{\prime}\right\rangle} \lambda\right)\right|(\bmod 2)$ whenever $m$ is odd.

(In the second of these conditions, we interpret $T_{1}^{(l+1)}$ as $\varnothing$ in type B.) Then, the projection in (4) is precisely the quotient by $\sim$. We can formulate one particular equivalence under $\sim$ quite easily, as follows. If we are working in type $B$, let $\tilde{S}_{1}=S_{1} \backslash\left\{j_{l}\right\}$, and note that this set has an even number of elements.

Lemma 3.2. Given a marked partition ${ }^{\langle\nu\rangle} \lambda \in \tilde{\mathcal{P}}_{X}(n)$, define

$$
\nu^{\prime}=T_{0}\left({ }^{\langle\nu\rangle} \lambda\right) \cup \begin{cases}S_{1}(\lambda) \backslash T_{1}\left({ }^{\langle\nu\rangle} \lambda\right) & \text { in types } C \text { and } D, \\ \left(\tilde{S}_{1}(\lambda) \backslash T_{1}\left({ }^{\langle\nu\rangle} \lambda\right)\right) \cup\left(T_{1}\left({ }^{\langle\nu\rangle} \lambda\right) \cap\left\{j_{l}\right\}\right) & \text { in type } B .\end{cases}
$$

Then ${ }^{\left\langle\nu^{\prime}\right\rangle} \lambda \sim{ }^{\langle\nu\rangle} \lambda$.

Proof. It is easy to see that condition (b) above is satisfied when we replace $T_{1}$ by its complement in $S_{1}$ in types $C$ and $D$. In type $B$, we need to be careful when $m=l$, because there is no $j_{l+1}$, but the same idea goes through if we take only take the complement of that portion of $T_{1}$ which meets $\tilde{S}_{1}$, as in the above formula.

Consider the set

$$
\tilde{\mathcal{P}}_{X}^{\circ}(n)=\left\{{ }^{\langle\nu\rangle} \lambda \in \tilde{\mathcal{P}}_{X}(n) \mid T_{1}^{(m+1)}=T_{0}^{(m)}=\varnothing \text { whenever } m \text { is odd }\right\},
$$

which we call the set of reduced marked partitions. It is easy to see that the restricted map $\tilde{\mathcal{P}}_{X}^{\circ}(n) \rightarrow \mathcal{N}_{o, \bar{c}}$ is a bijection. An alternate description of these sets is as follows. If $\lambda$ is of type $B$ (resp. $C, D$ ), let us call a part of $\lambda$ markable if it is odd (resp. even, odd) and has odd (resp. even, even) height. Then we have

$$
\tilde{\mathcal{P}}_{X}^{\circ}(n)=\left\{{ }^{\langle\nu\rangle} \lambda \in \tilde{\mathcal{P}}_{X}(n) \mid \nu \text { consists only of markable parts of } \lambda\right\} \text {. }
$$

We will speak of elements of $\tilde{\mathcal{P}}_{X}(n)$ as labels for elements of $\mathcal{N}_{\mathrm{o}, \mathrm{c}}$ and $\mathcal{N}_{\mathrm{o}, \overline{\mathrm{c}}}$, and of elements of $\tilde{\mathcal{P}}_{X}^{\circ}(n)$ as the reduced labels for elements of $\mathcal{N}_{\mathrm{o}, \overline{\mathrm{c}}}$. Every element of $\tilde{\mathcal{P}}_{X}(n)$ is $\sim$-equivalent to exactly one element of $\tilde{\mathcal{P}}_{X}^{\circ}(n)$. The process of passing to the reduced label can be described as follows. Given ${ }^{\langle\nu\rangle} \lambda$, we define a new marked partition ${ }^{\left\langle\nu^{\prime}\right\rangle} \lambda$, which is characterized as follows: we have

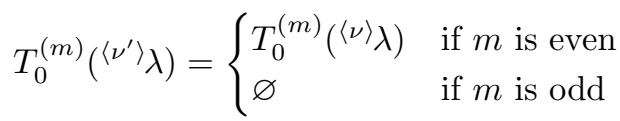

and

$$
T_{1}^{(m)}\left({ }^{\left\langle\nu^{\prime}\right\rangle} \lambda\right)= \begin{cases}\left\{j_{m}\right\} & \text { if } m \text { is odd and }\left|T_{1}^{(m+1)}\left({ }^{\langle\nu\rangle} \lambda\right) \cup T_{0}^{(m)}\left({ }^{\langle\nu\rangle} \lambda\right) \cup T_{1}^{(m)}\left({ }^{\langle\nu\rangle} \lambda\right)\right| \text { is odd } \\ \varnothing & \text { otherwise. }\end{cases}
$$

There is often a sensible way, given two arbitrary partitions $\lambda$ and $\nu$, to carry out a "reduction" procedure that generalizes the above one, even when ${ }^{\langle\nu\rangle} \lambda$ is not a valid marked partition. (This goes hand-in-hand with the idea of generalized height.) We must first fix one of the types $B, C$, or $D$ as the context in which we are working, but we do not require that $\lambda$ be a partition of that type. The only condition we impose is that when the context type is $B$ or $D, \nu$ must have an even number of parts. Note that the above definition of "markable part" makes sense without any restriction on $\lambda$, and let $m_{k}>\cdots>m_{1}$ be the set of markable parts of $\lambda$. We define $\nu^{\prime}$ by putting

$$
r_{\nu^{\prime}}\left(m_{i}\right)=\left\{\begin{array}{ll}
1 & \text { if } \mathrm{ht}_{\nu}\left(m_{i}\right)-\mathrm{ht}_{\nu}\left(m_{i+1}\right) \text { is odd, } \\
0 & \text { if } \mathrm{ht}_{\nu}\left(m_{i}\right)-\mathrm{ht}_{\nu}\left(m_{i+1}\right) \text { is even, }
\end{array} \quad r_{\nu^{\prime}}(a)=0 \text { if } a \text { is not a markable part of } \lambda,\right.
$$

where, when $i=k$, we interpret $\mathrm{ht}_{\nu}\left(m_{k+1}\right)$ as 0 . It is easy to verify that when ${ }^{\langle\nu\rangle} \lambda$ is a marked partition, this generalized procedure coincides with the above one for passing to a reduced marked partition. 
Let us return to the problem that unions and joins of marked partitions may not yield valid marked partitions. Typically, we employ the above procedure to pass from whatever partitions the formulas (2) and (3) yield to a reduced marked partition. Indeed, henceforth, unless explicitly stated otherwise, all marked partitions are assumed to be reduced, and if any possibly nonreduced marked partition appears in a formula, we silently assume that it is to be replaced by an equivalent reduced one.

3.5. Duality and special orbits. We now recall the formulas for $d_{\mathrm{LS}}, d_{\mathrm{BV}}$, and $d_{\mathrm{S}}$ in the classical groups.

\begin{tabular}{|c|c|c|c|}
\hline Type $B$ : & $d_{\mathrm{LS}}(\lambda)=\lambda_{B}^{*}$ & $d_{\mathrm{BV}}(\lambda)=\lambda^{-} C^{*}$ & $d_{\mathrm{S}}(\nu, \eta)=\left(\nu \cup \eta_{C}^{-}\right)^{*}{ }_{C}$ \\
\hline Гур & $d_{\mathrm{LS}}(\lambda)=\lambda_{C}^{*}$ & $d_{\mathrm{BV}}(\lambda)=\lambda^{+}{ }_{B}^{*}$ & $d_{\mathrm{S}}(\nu, \eta)=\left(\nu \cup \eta^{+}{ }^{+}\right)^{\prime}$ \\
\hline уре $I$ & $d_{\mathrm{LS}}(\lambda)=\lambda^{*}{ }_{D}$ & $d_{\mathrm{BV}}(\lambda)=\lambda_{D}^{*}$ & $d_{\mathrm{S}}(\nu, \eta)=\left(\nu \cup \eta^{*}\right.$ \\
\hline
\end{tabular}

The formulas for $d_{\mathrm{BV}}$ are obtained by combining the formulas for $d_{\mathrm{LS}}$ with the following formulas for the order-preserving bijection between $\mathcal{N}_{\mathrm{o}}^{\mathrm{sp}}$ and ${ }^{L} \mathcal{N}_{\mathrm{o}}^{\mathrm{sp}}$ in types $B$ and $C$ :

$$
\begin{array}{ll}
\mathcal{N}_{\mathrm{o}}^{\mathrm{sp}}\left(B_{n}\right) \rightarrow \mathcal{N}_{\mathrm{o}}^{\mathrm{sp}}\left(C_{n}\right): & \lambda \mapsto \lambda^{-}{ }_{C} \\
\mathcal{N}_{\mathrm{o}}^{\mathrm{sp}}\left(C_{n}\right) \rightarrow \mathcal{N}_{\mathrm{o}}^{\mathrm{sp}}\left(B_{n}\right): & \lambda \mapsto \lambda^{+}{ }_{B}
\end{array}
$$

In fact, these same formulas can be evaluated on nonspecial partitions in $\mathcal{N}_{\mathrm{o}}\left(B_{n}\right)$ and $\mathcal{N}_{\mathrm{o}}\left(C_{n}\right)$ : they then compute the following composition of maps:

$$
\mathcal{N}_{\mathrm{o}} \stackrel{d_{\mathrm{LS}}^{2}}{\longrightarrow} \mathcal{N}_{\mathrm{o}}^{\mathrm{sp}} \stackrel{\simeq}{\longrightarrow}{ }^{L} \mathcal{N}_{\mathrm{o}}^{\mathrm{sp}} .
$$

The formulas for $d_{\mathrm{S}}$ are given in [18]. We are now in a position to revisit the proof of Proposition 2.3.

Proof of Proposition 2.3 in the classical types. We need to show that $d_{\mathrm{S}}(\mathcal{O}, 1) \geq d_{\mathrm{S}}(\mathcal{O}, C)$; this should follow from a quick computation using the above formulas. We carry it out now in type $D$. Starting with $\lambda=\nu \cup \eta$, it is easy to see that

$$
\begin{aligned}
\eta & \leq \eta^{*}{ }^{*} \\
\lambda=\nu \cup \eta & \leq \nu \cup \eta^{*}{ }^{*} \\
\lambda^{*} & \geq\left(\nu \cup \eta^{*} D^{*}\right)^{*} \\
\lambda^{*}{ }_{D} & \geq\left(\nu \cup \eta^{*} D^{*}\right)^{*}{ }_{D} .
\end{aligned}
$$

Essentially the same reasoning works in types $B$ and $C$ as well, although we need to replace (6b) above with the following slightly less trivial inequalities:

$$
\lambda^{-}{ }_{C} \leq \nu \cup \eta^{-}{ }_{C}, \quad \lambda^{+}{ }_{B} \leq \nu \cup \eta^{+}{ }_{B} .
$$

Moreover, in types $B$ and $C$, we need to use the observations that $\lambda^{-} C^{*}=\lambda^{-} C^{*} C$ and $\lambda^{+} B^{*}=\lambda^{+} B^{*} B$, respectively, to pass from $(6 \mathrm{c})$ to $(6 \mathrm{~d})$.

We also recall the recipe for computing Sommers' canonical inverse. if $\lambda$ is of type $B$ (resp. $C$ or $D$ ), we let $\pi$ be the set of even (resp. odd) parts of $\lambda^{*}$ with odd multiplicity. Then the canonical inverse is given by ${ }^{\langle\pi\rangle} d_{\mathrm{BV}}(\lambda)$, where we pass to the reduced marked partition if necessary. (In [18], Sommers regards the canonical inverse as a map ${ }^{L} \mathcal{N}_{\mathrm{o}} \rightarrow \mathcal{N}_{\mathrm{o}, \mathrm{c}}$, so he made no comment about passing a reduced marked partition, but in the present context, we regard it as a map ${ }^{L} \mathcal{N}_{\mathrm{o}} \rightarrow \mathcal{N}_{\mathrm{o}, \overline{\mathrm{c}}}$.)

The images of $d_{\mathrm{LS}}$ and $d_{\mathrm{BV}}$ consist precisely of the set of special orbits, which are labelled by special partitions. A characterization of special partitions may be found in [9]. If $\lambda$ is a $B$ - (resp. $C$-, $D-$-) partition, it is special if all its even (resp. odd, even) parts have odd (resp. even, even) height. Moreover, if $\lambda$ is a special $B$ - (resp. $C$-) partition, then $\lambda^{*}$ is also a special $B$ - (resp $C$-) partition. If $\lambda$ is a special $D$-partition, then $\lambda^{*}$ is a (not necessarily special) $C$-partition. We conclude with a lemma about formulas for special partitions.

Lemma 3.3. The following identities hold: $\lambda^{-} C^{*}=\lambda^{*-}{ }_{C}$ for $\lambda \in \mathcal{P}_{B}(n), \lambda^{+}{ }_{B}{ }^{*}=\lambda^{*+}{ }_{B}$ for $\lambda \in \mathcal{P}_{C}(n)$, and $\lambda^{*}{ }^{*}=\lambda^{+-}{ }_{C}$ if either $\lambda \in \mathcal{P}_{D}(n)$ or $\lambda^{*} \in \mathcal{P}_{C}(n)$. 
Proof. The proof establishes all three formulas simultaneously by induction on the sum of the partition. One verifies it by direct calculation for the smallest partitions: [3] and $\left[1^{3}\right]$ in type $B,[2]$ and $\left[1^{2}\right]$ in type $C$, and $\left[1^{2}\right]$ in type $D$. We work out the inductive step when $\lambda$ is of type $B$; the others are handled similarly. Let $m=\# \lambda$, and let $b$ be the smallest part of $\lambda$. Note that $m$ is necessarily odd. We can write $\lambda=\left[b^{m}\right] \vee \lambda^{\prime}$, where $\lambda^{\prime}$ is a $B$-partition if $b$ is even, and a $C$-partition if $b$ is odd. Suppose first that $b$ is odd. We have $\lambda^{-}=\left[b^{m}\right]^{-} \vee \lambda^{\prime}$, so $\lambda^{-}{ }_{C}=\left[b^{m}\right]^{-}{ }_{C} \vee \lambda_{D}^{\prime}$ by Lemma 3.1. Now, $\left[b^{m}\right]^{-}{ }_{C}=\left[b^{m-1}, b-1\right]$, so we get $\lambda^{-} C^{*}=\left[m^{b-1}, m-1\right] \cup \lambda_{D}^{\prime *}$. Using the inductive hypothesis, we rewrite this as $\left[m^{b}\right]^{-} \cup \lambda^{\prime *+-}{ }_{C}$. Another appeal to Lemma 3.1 lets us conclude that this last expression is equal to $\lambda^{*-}{ }_{C}$. The case of $b$ even is handled similarly, as are types $C$ and $D$.

\section{Construction in the Classical Groups}

We are now ready to define the map $\bar{d}: \mathcal{N}_{\mathrm{o}, \overline{\mathrm{c}}} \rightarrow{ }^{L} \mathcal{N}_{\mathrm{o}, \overline{\mathrm{c}}}$. Since we know that we want $p r_{1} \circ \bar{d}$ to agree with $d_{\mathrm{S}}$, where $\operatorname{pr}_{1}: \mathcal{N}_{\mathrm{o}, \overline{\mathrm{c}}} \rightarrow \mathcal{N}_{\mathrm{o}}$ is projection to the first member, the main difficulty is defining the marking partition on the range. Given a reduced marked partition ${ }^{\langle\nu\rangle} \lambda$, write $\nu=\left[n_{l}>\cdots>n_{1}\right]$, and assume that $l$ is even by taking $n_{1}=0$ if necessary in type $C$. Define

$$
\hat{\nu}=\left[\mathrm{ht}_{\lambda}\left(n_{1}\right)-1>\cdots>\mathrm{ht}_{\lambda}\left(n_{l}\right)-1\right] .
$$

(If we are in type $C$ and $n_{1}=0$, we need to say what $h_{\lambda}(0)$ means. We want this quantity to be even, since markable parts are supposed to have even height in type $C$. We take it to be the smallest even number larger than $\# \lambda$.) Next, if ${ }^{\langle\nu\rangle} \lambda=(\nu, \eta)$ is a marked partition of type $B$ (resp. $C, D$ ), we define

$$
\pi=\left\{\text { even (resp. odd, odd) parts of } \eta^{*} \text { with odd multiplicity }\right\} \text {. }
$$

We regard this set as a partition, each of whose parts has multiplicity 1 . We then put

$$
\begin{aligned}
& \text { Type B: } \quad\langle\rho\rangle_{\tau}=\left\langle{ }^{\langle\varnothing\rangle{ }^{*} \vee}{ }^{\langle\pi\rangle} \eta^{-} C^{*} \quad \bar{d}\left({ }^{\langle\nu\rangle} \lambda\right)={ }^{\langle\hat{\nu} \cup \rho\rangle} \tau_{C}\right.
\end{aligned}
$$

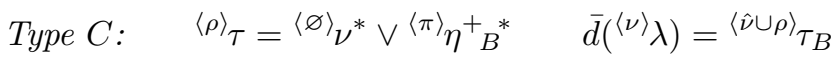

$$
\begin{aligned}
& \text { Type D: } \quad{ }^{\langle\rho\rangle_{\tau}}=\left\langle{ }^{\langle\varnothing\rangle{ }^{*} \vee}{ }^{\langle\pi\rangle} \eta^{*}{ }_{D} \quad \bar{d}\left({ }^{\langle\nu\rangle} \lambda\right)={ }^{\langle\hat{\nu} \cup \rho\rangle}{ }_{D} .\right.
\end{aligned}
$$

Computing with these formulas by hand can be quite cumbersome, especially since one must pass to a reduced marked partition several times. To help elucidate the matter, we now discuss in detail the steps involved in computing $\bar{d}$ in type $B$. First, we find the partition $\tilde{\eta}=\eta^{-} C^{*}$. Next, $\pi$ may not be a valid marking partition for $\tilde{\eta}$, or it may simply not be reduced: in any case, we reduce $\langle\pi\rangle \tilde{\eta}$ to obtain a reduced marked partition ${ }^{\left\langle\pi^{\prime}\right\rangle} \tilde{\eta}$. Now, $\tau$ is simply the join $\nu^{*} \vee \tilde{\eta}$. To compute $\rho$, we must refer to the description of joins of marked partitions in Section 3: we first take $\rho^{\prime}$ to be the set of parts of $\tau$ whose heights are the same as the heights of the parts of $\pi$ in $\tilde{\eta}$. Then, $\langle\rho\rangle_{\tau}$ is the reduced marked partition obtained by reducing $\left\langle\rho^{\prime}\right\rangle_{\tau}$. Finally, let $\tilde{\tau}$ be the collapse $\tau_{C}$. Again, $\hat{\nu} \cup \rho$ may not be a valid reduced marking partition for $\tilde{\tau}$, but reducing $\langle\hat{\nu} \cup \rho\rangle \tilde{\mathcal{\tau}}$ will give us some ${ }^{\langle\sigma\rangle} \tilde{\tau}$. This is the final answer: $\bar{d}\left({ }^{\langle\nu\rangle} \lambda\right)=\langle\sigma\rangle \tilde{\tau}$. The following example illustrates this procedure.

Example 4.1. Consider the orbit $\mathcal{O}$ labelled by $\left[7,5,4^{2}, 3,2^{2}, 1^{2}\right]$ in type $B_{14}$, or $\mathfrak{s o}(29)$. This partition has three markable parts: 7,3 , and 1 . Therefore, $\bar{A}(\mathcal{O}) \simeq(\mathbb{Z} / 2 \mathbb{Z})^{2}$; the four possible marking partitions are $\varnothing,[3,1],[7,3]$, and $[7,1]$. Let us consider the conjugacy class corresponding to $[3,1]$. Writing $\langle[3,1]\rangle\left[7,5,4^{2}, 3,2^{2}, 1^{2}\right]$ as a pair, we have $(\nu, \eta)=\left([3,1],\left[7,5,4^{2}, 2^{2}, 1\right]\right)$. We compute $\eta^{-}{ }_{C}=\left[6^{2}, 4^{2}, 2^{2}\right]$, which is self-dual: $\eta^{-} C^{*}=\left[6^{2}, 4^{2}, 2^{2}\right]$. We have $\nu^{*}=\left[2,1^{2}\right]$, so $\tau=\left[8,7,5,4,2^{2}\right]$. Finally, $\tau_{C}=\left[8,6^{2}, 4,2^{2}\right]$.

To compute the marking partition, we have $\eta^{*}=\left[7,6,4^{2}, 2,1^{2}\right]$, so $\pi=[6,2]$. Both parts of $\pi$ are markable in $\eta^{-} C^{*}$, so $\langle\pi\rangle \eta^{-} C^{*}$ is already reduced. Taking the join with $\nu^{*}$ yields ${ }^{\langle[7,2]\rangle}\left[8,7,5,4,2^{2}\right]$, which becomes $\langle[4,2]\rangle\left[8,7,5,4,2^{2}\right]$ when we reduce it. Finally, $\hat{\nu}=[8,4]$, so for the final answer, we take the reduced marked partition corresponding to $\left\langle\left[8,4^{2}, 2\right]\right\rangle\left[8,6^{2}, 4,2^{2}\right]$, arriving at

$$
\bar{d}\left({ }^{\langle[3,1]\rangle}\left[7,5,4^{2}, 3,2^{2}, 1^{2}\right]\right)=\langle[4,2]\rangle\left[8,6^{2}, 4,2^{2}\right] .
$$

In this and the following section, we will be ensconced in many laborious computations with the above formulas. Most of the results must actually be proved thrice, once in each of types $B, C$, and $D$; but we will usually only write out the full details in type $B$, and just make cursory remarks about the nature of the calculations in the other types. 
We can establish the following two properties of $\bar{d}$ immediately from the definition. Once again, $p r_{1}$ : $\mathcal{N}_{\mathrm{o}, \overline{\mathrm{c}}} \rightarrow \mathcal{N}_{\mathrm{o}}$ is the obvious projection map. Let us also recall Sommers' canonical inverse, which was mentioned near the end of Section 2. This is a certain right inverse to $d_{\mathrm{S}}$; see [18] for its construction.

Proposition 4.2. We have that $\mathrm{pr}_{1} \circ \bar{d}$ agrees with $d_{\mathrm{S}}$.

Proof. In type $B$, the underlying partition of $\bar{d}(\langle\nu\rangle)$ is $\tau_{C}=\left(\nu^{*} \vee \eta^{-} C^{*}\right)_{C}=\left(\nu \cup \eta^{-}{ }^{*}\right)_{C}$, which is precisely the formula for $d_{\mathrm{S}}(\nu, \eta)$ in type $B$. Types $C$ and $D$ are equally easy to handle.

Proposition 4.3. Given an orbit $\mathcal{O}$ labelled by a partition $\lambda$, the conjugacy class labelled by $\bar{d}\left({ }^{(\varnothing)} \lambda\right)$ coincides with Sommers' canonical inverse for $\mathcal{O}$.

Proof. When $\nu=\varnothing$ and $\eta=\lambda$, the formula for $\langle\rho\rangle_{\tau}$ in (9) agrees with Sommers' recipe for the canonical inverse. At this stage, $\tau=d_{\mathrm{BV}}(\lambda)$ is already a $C$-, $B$-, or $D$-partition (in types $B, C$, and $D$, respectively), so the additional collapse of $\tau$ in the formula for $\bar{d}$ does nothing. We also have $\hat{\nu}=\varnothing$, so $\bar{d}\left({ }^{\langle\nu\rangle} \lambda\right)={ }^{\langle\pi\rangle} \tau$.

Before we can set about proving that the above map is, in fact, an extended duality map as defined in Section 1, we need to develop some techniques for manipulating marked partitions. The formulas we have so far are too opaque to be tackled in their raw form when we want to prove things about them. We spend the rest of the section showing how to break down a marked partition into "blocks," and how to compute $\bar{d}$ piecemeal on the individual blocks.

Lemma 4.4. Suppose that ${ }^{\langle\nu\rangle} \lambda=\langle\varnothing\rangle\left[a^{l}\right] \vee\left\langle\nu^{\prime}\right\rangle \lambda^{\prime}$, where $\lambda$ has l parts.

(a) If ${ }^{\langle\nu\rangle} \lambda$ is of type $B$ or $D$ and $a$ is even, then $\bar{d}\left({ }^{\langle\nu\rangle} \lambda\right)=\left\langle{ }^{\langle\varnothing\rangle}\left[{ }^{a}\right] \cup \bar{d}\left({ }^{\left\langle\nu^{\prime}\right\rangle} \lambda^{\prime}\right)\right.$. In this case, ${ }^{\left\langle\nu^{\prime}\right\rangle} \lambda^{\prime}$ is of the same type as ${ }^{\langle\nu\rangle} \lambda$.

(b) If ${ }^{\langle\nu\rangle} \lambda$ is of type $C$, a is odd, and $l$ is even, then $\bar{d}\left({ }^{\langle\nu\rangle} \lambda\right)=\langle\varnothing\rangle\left[l+1, l^{a-1}\right] \cup \bar{d}\left({ }^{\left\langle\nu^{\prime}\right\rangle} \lambda^{\prime}\right)$. Here, ${ }^{\left\langle\nu^{\prime}\right\rangle} \lambda^{\prime}$ is of type $D$.

In both cases, we also have $\bar{d}^{2}\left({ }^{\langle\nu\rangle} \lambda\right)={ }^{\langle\varnothing\rangle}\left[a^{l}\right] \vee \bar{d}^{2}\left(\left\langle\nu^{\prime}\right\rangle \lambda^{\prime}\right)$.

Proof. Let us write ${ }^{\langle\nu\rangle} \lambda=(\nu, \eta)$ and ${ }^{\left\langle\nu^{\prime}\right\rangle} \lambda^{\prime}=\left(\nu^{\prime}, \eta^{\prime}\right)$, and let us refer back to the formulas for $d_{\mathrm{S}}$. If $\eta$ has $n$ parts, then

$$
\nu=\left[a^{l-n}\right] \vee \nu^{\prime} \quad \text { and } \quad \eta=\left[a^{n}\right] \vee \eta^{\prime} .
$$

We will prove part (a) when ${ }^{\langle\nu\rangle} \lambda$ is of type $B$. The type- $D$ case of part (a), as well as part (b) and the statement for $\bar{d}^{2}$, are handled similarly. Now, $\eta^{-}$may be given by either $\left[a^{n}\right] \vee \eta^{\prime-}$ or $\left[a^{n-1}, a-1\right] \vee \eta^{\prime}$, depending on whether $\eta^{\prime}$ has $n$ parts or fewer than $n$ parts. We compute $\eta^{-}{ }_{C}$ with the appropriate formula from Lemma 3.1, and see that in either case, we get $\left[a^{n}\right] \vee \eta^{\prime-}{ }_{C}$ (possibly using the fact that $\left[a^{n-1}, a-1\right]^{+}{ }_{C}=$ $\left.\left[a^{n}\right]\right)$. Therefore,

$$
\nu \cup \eta^{-}{ }_{C}=\left(\left[a^{l-n}\right] \vee \nu^{\prime}\right) \cup\left(\left[a^{n}\right] \vee \eta^{\prime-}{ }_{C}\right)=\left[a^{l}\right] \vee\left(\nu^{\prime} \cup \eta^{\prime-}{ }_{C}\right) .
$$

Taking the transpose of both sides, we get

$$
\left(\nu \cup \eta^{-}{ }^{*}\right)^{*}=\left[l^{a}\right] \cup\left(\nu^{\prime} \cup \eta^{\prime-}{ }_{C}\right)^{*} .
$$

Now, we use another formula from Lemma 3.1 to compute the $C$-collapse of this expression. We obtain

$$
d_{\mathrm{S}}\left({ }^{\langle\nu\rangle} \lambda\right)=\left(\nu \cup \eta^{-}{ }_{C}\right)_{C}=\left[l^{a}\right] \cup\left(\nu^{\prime} \cup \eta^{\prime-}{ }_{C}\right)_{C}=\left[l^{a}\right] \cup d_{\mathrm{S}}\left({ }^{\left\langle\nu^{\prime}\right\rangle} \lambda^{\prime}\right) .
$$

We now need to compute the marking partition. If $\pi$ is defined from $\eta$ according to (8), and $\pi^{\prime}$ is defined analagously from $\eta^{\prime}$, we evidently have $\pi=\pi^{\prime}$. It is then easy to work through the formulas of (9) and see that $\rho=\rho^{\prime}$ as well, and finally that $\bar{d}\left({ }^{\langle\nu\rangle} \lambda\right)$ and $\bar{d}\left({ }^{\left\langle\nu^{\prime}\right\rangle} \lambda^{\prime}\right)$ have the same marking partition.

Lemma 4.5. Given ${ }^{\langle\nu\rangle} \lambda \in \tilde{\mathcal{P}}_{B}(n)$, suppose that ${ }^{\langle\nu\rangle} \lambda=\left\langle\nu_{1}\right\rangle \lambda_{1} \cup{ }^{\left\langle\nu_{2}\right\rangle} \lambda_{2}$, with ${ }^{\left\langle\nu_{1}\right\rangle} \lambda_{1} \in \tilde{\mathcal{P}}_{B}(m)$ and ${ }^{\left\langle\nu_{2}\right\rangle} \lambda_{2} \in$ $\tilde{\mathcal{P}}_{D}(n-m)$. Suppose furthermore that $\lambda_{1}$ is evenly superior to $\lambda_{2}$. Then $\bar{d}\left({ }^{\langle\nu\rangle} \lambda\right)=\bar{d}\left({ }^{\left\langle\nu_{1}\right\rangle} \lambda_{1}\right) \vee \bar{d}\left({ }^{\left\langle\nu_{2}\right\rangle} \lambda_{2}\right)$. (Note that the term $\bar{d}\left(\left\langle\nu_{2}\right\rangle \lambda_{2}\right)$ is to be computed in type D.)

Proof. Write ${ }^{\langle\nu\rangle} \lambda=(\nu, \eta),{ }^{\left\langle\nu_{1}\right\rangle} \lambda_{1}=\left(\nu_{1}, \eta_{1}\right)$, and ${ }^{\left\langle\nu_{2}\right\rangle} \lambda_{2}=\left(\nu_{2}, \eta_{2}\right)$ in the notation of pairs, and let $\pi, \pi_{1}$, and $\pi_{2}$ be the corresponding partitions as defined in (8). Note that $\eta_{1}$ has an odd number of parts, and $\left|\eta_{1}\right|$ is odd, both because $\eta_{1}$ is a $B$-partition. We consult Lemma 3.1, starting with $\eta^{-}=\eta_{1} \cup \eta_{2}^{-}$, and find that $\eta^{-}{ }_{C}=\eta_{1}^{-} C \cup \eta_{2}^{-+}{ }_{C}$; then, Lemma 3.3 tells us that we actually have $\eta^{-}{ }_{C}=\eta_{1}^{-} C \cup \eta_{2}^{*} D^{*}$. Because $\eta_{1}$ has an 
odd number of parts, even parts of $\eta^{*}$ correspond to odd parts of $\eta_{2}^{*}$, so $\pi_{1}$ and $\pi_{2}$ are related to $\pi$ as in the following equation:

$$
\langle\pi\rangle \eta^{-} C^{*}=\left\langle\pi_{1}\right\rangle \eta_{1}^{-} C^{*} \vee\left\langle\pi_{2}\right\rangle \eta_{2}^{*} D
$$

Since $\nu=\nu_{1} \cup \nu_{2}$, we have $\nu^{*}=\nu_{1}^{*} \vee \nu_{2}^{*}$; it follows directly that

$$
\langle\varnothing\rangle \nu^{*} \vee\langle\pi\rangle \eta^{-} C^{*}=\left({ }^{\langle\varnothing\rangle} \nu_{1}^{*} \vee\left\langle\pi_{1}\right\rangle \eta_{1}^{-} C^{*}\right) \vee\left({ }^{\langle\varnothing\rangle} \nu_{2}^{*} \vee^{\left\langle\pi_{2}\right\rangle} \eta_{2}^{*} D\right)
$$

Write this equation, following (9), as ${ }^{\langle\rho\rangle} \tau=\left\langle\rho_{1}\right\rangle_{1} \vee\left\langle\rho_{2}\right\rangle_{\tau_{2}}$. Now, $\tau_{1}$ has an odd number of columns, because $\# \lambda_{1}$ is odd. (One might worry that it could have fewer columns due to the "-" operation, if the smallest part of $\eta_{1}$ were 1 , but that is not possible since $\lambda_{1}$ is superior to $\lambda_{2}$.) Now we make use of the hypothesis of even superiority: another appeal to Lemma 3.1 tells us exactly that $\tau_{C}=\tau_{1 C} \vee \tau_{2 D}$. At this point, we have established that

$$
d_{\mathrm{S}}\left({ }^{\langle\nu\rangle} \lambda\right)=d_{\mathrm{S}}\left({ }^{\left\langle\nu_{1}\right\rangle} \lambda_{1}\right) \vee d_{\mathrm{S}}\left({ }^{\left\langle\nu_{2}\right\rangle} \lambda_{2}\right)
$$

Now consider the marking partition. If $n$ is a part of $\nu_{2}$, we have ht $\lambda_{\lambda}(n)=h_{\lambda_{2}}(n)+\# \lambda_{1}$. We therefore have $\hat{\nu}=\hat{\nu}_{1} \cup\left(\left[\left(\# \lambda_{1}\right)^{\# \hat{\nu}_{2}}\right] \vee \hat{\nu}_{2}\right)$. Combining this description with (12) and (11), we find

$$
\langle\hat{\nu} \cup \rho\rangle_{\tau_{C}}=\left\langle\hat{\nu}_{1} \cup \rho_{1}\right\rangle_{\tau_{1 C}} \vee\left\langle\hat{\nu}_{2} \cup \rho_{2}\right\rangle_{\tau_{2 D}}
$$

as desired.

Entirely analagous arguments establish the three cases of the following lemma.

Lemma 4.6. Let ${ }^{\langle\nu\rangle} \lambda$ be a marked partition, and suppose that ${ }^{\langle\nu\rangle} \lambda=\left\langle\nu_{1}\right\rangle_{1} \cup\left\langle\nu_{2}\right\rangle \lambda_{2}$.

(a) If ${ }^{\langle\nu\rangle} \lambda \in \tilde{\mathcal{P}}_{C}(n)$, let us also suppose that ${ }^{\left\langle\nu_{1}\right\rangle} \lambda_{1} \in \tilde{\mathcal{P}}_{C}(m)$, that ${ }^{\left\langle\nu_{2}\right\rangle} \lambda_{2} \in \tilde{\mathcal{P}}_{C}(n-m)$, that $\lambda_{1}$ and $\nu_{1}$ have an even number of parts, and that $\lambda_{1}$ is oddly superior to $\lambda_{2}$. Then $\bar{d}\left({ }^{\langle\nu\rangle} \lambda\right)=\bar{d}\left({ }^{\left\langle\nu_{1}\right\rangle} \lambda_{1}\right)-\vee \bar{d}\left({ }^{\left\langle\nu_{2}\right\rangle} \lambda_{2}\right)$. Here, $\bar{d}\left({ }^{\left\langle\nu_{1}\right\rangle} \lambda_{1}\right)_{-}$is to be understood as applying the _ operation to the underlying partition. The largest part of the underlying partition is odd, so the marking partition is unaffected.

(b) If ${ }^{\langle\nu\rangle} \lambda \in \tilde{\mathcal{P}}_{D}(n)$, let us also suppose that ${ }^{\left\langle\nu_{1}\right\rangle} \lambda_{1} \in \tilde{\mathcal{P}}_{D}(m)$, that ${ }^{\left\langle\nu_{2}\right\rangle} \lambda_{2} \in \tilde{\mathcal{P}}_{D}(n-m)$, and that $\lambda_{1}$ is evenly superior to $\lambda_{2}$. Then $\bar{d}\left({ }^{\langle\nu\rangle} \lambda\right)=\bar{d}\left({ }^{\left\langle\nu_{1}\right\rangle} \lambda_{1}\right) \vee \bar{d}\left({ }^{\left\langle\nu_{2}\right\rangle} \lambda_{2}\right)$.

(c) In the context of case (a), let us further suppose that $\lambda, \nu, \lambda_{2}$, and $\nu_{2}$ have even numbers of parts. Then $\bar{d}\left({ }^{\langle\nu\rangle} \lambda\right)_{-}=\bar{d}\left(\left\langle\nu_{1}\right\rangle \lambda_{1}\right)_{-} \vee \bar{d}\left(\left\langle\nu_{2}\right\rangle \lambda_{2}\right)_{-}$.

Case (c) of this lemma may seem bizarre, but we will arrive at a use for it shortly.

Returning to the context of Lemma 4.5 , let $m$ be the even number arising in the definition of "evenly superior" for $\lambda_{1}$ and $\lambda_{2}$, and let $l=\# \lambda_{1}$. (Note that $l$ is odd.) There is marked $B$-partition $\left\langle\nu_{1}^{\prime}\right\rangle \lambda_{1}^{\prime}$ such that ${ }^{\left\langle\nu_{1}\right\rangle} \lambda_{1}=\langle\varnothing\rangle\left[m^{l}\right] \vee\left\langle\nu_{1}^{\prime}\right\rangle \lambda_{1}^{\prime}$. Using Lemma 4.4, we can write

$$
\begin{aligned}
\bar{d}\left({ }^{\langle\nu\rangle} \lambda\right) & =\left({ }^{\langle\varnothing\rangle}\left[l^{m}\right] \cup \bar{d}\left({ }^{\left\langle\nu_{1}^{\prime}\right\rangle} \lambda_{1}^{\prime}\right)\right) \vee \bar{d}\left({ }^{\left\langle\nu_{2}\right\rangle} \lambda_{2}\right) \\
& =\left({ }^{\langle\varnothing\rangle}\left[l^{m}\right] \vee \bar{d}\left({ }^{\left\langle\nu_{2}\right\rangle} \lambda_{2}\right)\right) \cup \bar{d}\left({ }^{\left\langle\nu_{1}^{\prime}\right\rangle} \lambda_{1}^{\prime}\right),
\end{aligned}
$$

where we have made use of the fact that the largest part of $\lambda_{2}$ is at most $m$, so the underlying partition of $\bar{d}\left({ }^{\left\langle\nu_{2}\right\rangle} \lambda_{2}\right)$ has at most $m$ parts. We can apply part (b) of Lemma 4.4 to the first term and write

$$
\bar{d}\left({ }^{\langle\varnothing\rangle}\left[l^{m}\right] \vee \bar{d}\left({ }^{\left\langle\nu_{2}\right\rangle} \lambda_{2}\right)\right)={ }^{\langle\varnothing\rangle}\left[m+1, m^{l-1}\right] \cup \bar{d}^{2}\left({ }^{\left\langle\nu_{2}\right\rangle} \lambda_{2}\right) .
$$

Now, the union in (13) is exactly of the form demanded by part (a) of Lemma 4.6, so we can apply that statement here.

$$
\begin{aligned}
\bar{d}^{2}\left({ }^{\langle\nu\rangle} \lambda\right) & =\bar{d}\left({ }^{\langle\varnothing\rangle}\left[l^{m}\right] \vee \bar{d}\left({ }^{\left\langle\nu_{2}\right\rangle} \lambda_{2}\right)\right)-\vee \bar{d}^{2}\left({ }^{\left\langle\nu_{1}^{\prime}\right\rangle} \lambda_{1}^{\prime}\right) \\
& =\left({ }^{\langle\varnothing\rangle}\left[m^{l}\right] \cup \bar{d}^{2}\left({ }^{\left\langle\nu_{2}\right\rangle} \lambda_{2}\right)\right) \vee \bar{d}^{2}\left({ }^{\left\langle\nu_{1}^{\prime}\right\rangle} \lambda_{1}^{\prime}\right) \\
& =\left({ }^{\langle\varnothing\rangle}\left[m^{l}\right] \vee \bar{d}^{2}\left({ }^{\left\langle\nu_{1}^{\prime}\right\rangle} \lambda_{1}^{\prime}\right)\right) \cup \bar{d}^{2}\left({ }^{\left\langle\nu_{2}\right\rangle} \lambda_{2}\right)
\end{aligned}
$$

By one final application of Lemma 4.4, we obtain the following result for type $B$. Similar calculations establish it in types $C$ and $D$.

Lemma 4.7. Let ${ }^{\langle\nu\rangle} \lambda={ }^{\left\langle\nu_{1}\right\rangle} \lambda_{1} \cup{ }^{\left\langle\nu_{2}\right\rangle} \lambda_{2}$ be a decomposition as in Lemma 4.5 or 4.6. Then $\bar{d}^{2}\left({ }^{\langle\nu\rangle} \lambda\right)=$ $\bar{d}^{2}\left({ }^{\left\langle\nu_{1}\right\rangle} \lambda_{1}\right) \cup \bar{d}^{2}\left({ }^{\left\langle\nu_{2}\right\rangle} \lambda_{2}\right)$. 
Now, we can use Lemma 4.6(b) iteratively to split up a marked $D$-partition into smaller and smaller pieces. We can also do the same in type $B$, if we use Lemma 4.5 and Lemma 4.6(b) in combination. Similarly, parts (a) and (c) of Lemma 4.6 taken together let us split up $C$-partitions into smaller and smaller pieces. The following definition captures the precise nature of the permitted decompositions.

Definition 4.8. Suppose we have ${ }^{\langle\nu\rangle} \lambda={ }^{\left\langle\nu_{1}\right\rangle} \lambda_{1} \cup \cdots \cup\left\langle\nu_{k}\right\rangle \lambda_{k}$. Such a decomposition is called a division into blocks of ${ }^{\langle\nu\rangle} \lambda$, and each ${ }^{\left\langle{ }^{i}\right\rangle_{i}} \lambda_{i}$ is called a block, under the circumstances described below.

If ${ }^{\langle\nu\rangle} \lambda \in \tilde{\mathcal{P}}_{B}(n)$, we require that ${ }^{\left\langle\nu_{1}\right\rangle} \lambda_{1} \in \tilde{\mathcal{P}}_{B}\left(k_{1}\right)$ and that ${ }^{\left\langle\nu_{i}\right\rangle} \lambda_{i} \in \tilde{\mathcal{P}}_{D}\left(k_{i}\right)$ for $i>1$. Furthermore, $\lambda_{i}$ must be evenly superior to $\lambda_{i+1}$ for $i=1, \ldots, k-1$.

If ${ }^{\langle\nu\rangle} \lambda \in \tilde{\mathcal{P}}_{C}(n)$, we require that ${ }^{\left\langle\nu_{i}\right\rangle} \lambda_{i} \in \tilde{\mathcal{P}}_{C}\left(k_{i}\right)$ for all $i$, and that $\lambda_{i}$ and $\nu_{i}$ have an even number of parts for $i=1, \ldots, k-1$. Furthermore, $\lambda_{i}$ must be oddly superior to $\lambda_{i+1}$ for $i=1, \ldots, k-1$.

If ${ }^{\langle\nu\rangle} \lambda \in \tilde{\mathcal{P}}_{D}(n)$, we require that ${ }^{\left\langle\nu_{i}\right\rangle} \lambda_{i} \in \tilde{\mathcal{P}}_{D}(m)$ for all $i$. Furthermore, $\lambda_{i}$ must be evenly superior to $\lambda_{i+1}$ for $i=1, \ldots, k-1$.

We now combine Lemmas 4.5, 4.6, and 4.7 to obtain the following concise statement.

Proposition 4.9. Let $\left.{ }^{\langle\nu\rangle} \lambda={ }^{\left\langle{ }_{1}\right\rangle}\right\rangle_{1} \cup \cdots \cup{ }^{\left\langle\nu_{k}\right\rangle} \lambda_{k}$ be a division into blocks. Then, $\bar{d}$ can be computed as follows:

$$
\begin{aligned}
& \text { Type B: } \quad \bar{d}\left({ }^{\langle\nu\rangle} \lambda\right)=\bar{d}\left({ }^{\left\langle\nu_{1}\right\rangle} \lambda_{1}\right) \vee \cdots \vee \bar{d}\left({ }^{\left\langle\nu_{k}\right\rangle} \lambda_{k}\right) \\
& \text { Type } C: \quad \bar{d}\left({ }^{\langle\nu\rangle} \lambda\right)=\bar{d}\left({ }^{\left\langle\nu_{1}\right\rangle} \lambda_{1}\right)_{-} \vee \cdots \vee \bar{d}\left({ }^{\left\langle\nu_{k-1}\right\rangle} \lambda_{k-1}\right)_{-} \vee \bar{d}\left({ }^{\left\langle\nu_{k}\right\rangle} \lambda_{k}\right) \\
& \text { Type D: } \quad \bar{d}\left({ }^{\langle\nu\rangle} \lambda\right)=\bar{d}\left({ }^{\left\langle\nu_{1}\right\rangle} \lambda_{1}\right) \vee \cdots \vee \bar{d}\left({ }^{\left\langle\nu_{k}\right\rangle} \lambda_{k}\right) \\
& \text { Moreover, in all types, } \bar{d}^{2}\left({ }^{\langle\nu\rangle} \lambda\right)=\bar{d}^{2}\left({ }^{\left\langle\nu_{1}\right\rangle} \lambda_{1}\right) \cup \cdots \cup \bar{d}^{2}\left({ }^{\left\langle\nu_{k}\right\rangle} \lambda_{k}\right) \text {. }
\end{aligned}
$$

The motivation for developing the idea of divisions into blocks is our hope that we can cut up arbitrary marked partitions into blocks that are very simple in some sense, and that such blocks will be easy to work with when we set about the task of proving the main theorems. We now state precisely the sort of blocks we hope to obtain.

Definition 4.10. A basic block of type $B$ (resp. $C, D$ ) is a marked partition ${ }^{\langle\nu\rangle} \lambda$ such that $\nu$ has one or two parts, say $\left[n_{2}\right]$ or $\left[n_{2}>n_{1}\right]$, such that $n_{1}$ (if it exists) is the smallest part of $\lambda$, and such that $n_{2}$ is the largest part of odd (resp. even, even) height in $\lambda$. The circumstance of $\nu$ having only one part can occur only in type $C$; in this case, we often regard $\nu$ as having two parts by putting $n_{1}=0$. A basic block is called ultrabasic if it meets the additional condition that $n_{1} \leq 1$.

Proposition 4.11. Any marked partition has a division into blocks such that each block is either a trivially marked partition or a basic block.

Proof. This is easily seen by induction on the number of parts of the underlying partition. Given a marked partition ${ }^{\langle\nu\rangle} \lambda$ of type $B$, let $a$ be the first part of odd height. If $a$ is even (and therefore unmarkable) or odd and unmarked, we put $\lambda_{1}=\chi_{\mathrm{ht}(a)}^{+}(\lambda)$ and $\lambda_{2}=\chi_{\mathrm{ht}(a)}^{-}(\lambda)$. Then, ${ }^{\langle\nu\rangle} \lambda=\langle\varnothing\rangle \lambda_{1} \cup{ }^{\langle\nu\rangle} \lambda_{2}$ is a division into blocks in which the first term is trivially marked, and in the second, $\lambda_{2}$ has fewer parts than $\lambda$.

If $a$ is a marked part, let $b$ be the second marked part. This time we take $\lambda_{1}=\chi_{\mathrm{ht}(b)}^{+}(\lambda)$ and $\lambda_{2}=\chi_{\mathrm{ht}(b)}^{-}(\lambda)$. This time, ${ }^{\langle\nu\rangle} \lambda=\langle[a>b]\rangle \lambda_{1} \cup\langle\nu \backslash\{a, b\}\rangle \lambda_{2}$ is a division into blocks whose first term is a basic block. Similar arguments work in types $C$ and $D$.

Henceforth, all our arguments regarding properties of $\bar{d}$ will address only basic and trivially marked blocks.

Proposition 4.12. If ${ }^{\langle\nu\rangle} \lambda$ is a basic block, then $d_{\mathrm{S}}\left({ }^{\langle\nu\rangle} \lambda\right)$ can be computed by the following simplified formulas:

$$
\begin{array}{ll}
\text { Type B: } & d_{\mathrm{S}}\left({ }^{\langle\nu\rangle} \lambda\right)=\lambda^{-*}{ }_{C} \\
\text { Type } C: & d_{\mathrm{S}}\left({ }^{\langle\nu\rangle} \lambda\right)=\lambda^{+*}{ }_{B} \\
\text { Type D: } & d_{\mathrm{S}}\left({ }^{\langle\nu\rangle} \lambda\right)=\lambda^{+-*}{ }_{D}
\end{array}
$$

Proof. If ${ }^{\langle\nu\rangle} \lambda$ is a basic block, write $\nu=\left[n_{2}>n_{1}\right]$. Let us assume for the time being that ${ }^{\langle\nu\rangle} \lambda$ is an ultrabasic block. This will make our calculations less cumbersome. We will obtain a formula; then, at the end of the proof, we use Lemma 4.4 to see that the same formula holds for general basic blocks. 
Suppose we are working in type $B$, so $n_{1}=1$. Let $h_{i}=\mathrm{ht}_{\lambda}\left(n_{i}\right)$ for $i=1,2$. Thus $h_{1}$ is the total number of parts of $\lambda$. Let $\mu_{1}=\chi_{h_{2}-1}^{+}(\lambda)$ and $\mu_{2}=\chi_{h_{2}-1}^{-}(\lambda)$. Note that $\mu_{1}$ has only parts of even height, and that $r_{\mu_{2}}\left(n_{2}\right)=1$. Let $\mu_{2}^{\prime}$ be the partition gotten from $\mu_{2}$ by decreasing the multiplicities of $n_{2}$ and $n_{1}$ each by 1 . Since $n_{1}=1$, we have $\mu_{2}=\left[n_{2}\right] \cup \mu_{2+}^{\prime}$. Writing ${ }^{\langle\nu\rangle} \lambda$ as a pair $(\nu, \eta)$, we have $\eta=\mu_{1} \cup \mu_{2}^{\prime}$, and $\eta^{-}=\mu_{1} \cup \mu_{2}^{\prime}{ }^{-}$. Using Lemma 3.1, we get $\eta^{-}{ }_{C}=\mu_{1 C} \cup \mu_{2}^{\prime}{ }_{C}$, but since all parts of $\mu_{1}$ have even height, they all have even multiplicity, so $\mu_{1 C}=\mu_{1}$ :

$$
\eta^{-}{ }_{C}=\mu_{1} \cup \mu_{2}^{\prime-}{ }_{C} .
$$

Next, again using that $n_{1}=1$, we have $\nu \cup \eta^{-}{ }_{C}=\mu_{1} \cup\left[n_{2}\right] \cup \mu_{2}^{\prime-}{ }_{C+}$, or

$$
\left(\nu \cup \eta^{-}{ }^{-}\right)^{*}=\mu_{1}^{*} \vee\left[n_{2}\right]^{*} \vee \mu_{2}^{\prime-} C^{*+} .
$$

We use Lemma 3.1 to get $\left(\nu \cup \eta^{-}{ }_{C}\right)_{C}{ }_{C}=\mu_{1}^{*} \vee\left(\left[n_{2}\right]^{*} \vee \mu_{2}^{\prime}{ }^{-}{ }^{*+}\right)_{C}$. Since $\mu_{1}$ only has parts of even height, $\mu_{1}^{*}$ only has even parts, so the $C$-collapse does nothing. Using Lemma 3.1 yet again, we find that the second term is equal to $\left[n_{2}\right]^{*} \vee \mu_{2}^{\prime}{ }_{C} C^{*+}{ }_{B}$. Now $\mu_{2}^{\prime}$ is a $B$-partition, so Spaltenstein's formulas give us that $\mu_{2}^{\prime} C^{*+}{ }_{B}=\mu_{2}^{\prime *} B$. Finally, using the fact that $\left[n_{2}\right]^{*} \vee \mu_{2}^{\prime *}=\mu_{2}^{-*}$, we obtain $\left[n_{2}\right]^{*} \vee \mu_{2}^{\prime *} B=\mu_{2}^{-*} C$ :

$$
\left(\nu \cup \eta^{-}\right)^{*}{ }_{C}=\mu_{1}^{*} \vee \mu_{2}^{-*}{ }_{C} .
$$

Now, we know $\lambda^{-*}=\mu_{1}^{*} \vee \mu_{2}^{-*}$, and Lemma 3.1 would tell us that $\lambda^{-*}{ }_{C}=\mu_{1}^{*} \vee \vee \mu_{2}^{-*} C$. But since $\mu_{1}$ only has parts of even height, $\mu_{1}^{*}$ only has even parts, and the $C$-collapse does nothing do it. Thus (16) is given by $\lambda^{-*}$, as desired.

We do not give the details in types $C$ and $D$, but as an aid to those who wish to work them out, we list the analogues of (14), (15), and (16) here.

\section{Type $C$}

$$
\begin{aligned}
& {\left[n_{2}\right] \cup \eta^{+}{ }_{B}=\mu_{1} \cup \mu_{2}^{+} B-} \\
& \left(\nu \cup \eta^{+}{ }_{B}\right)^{*}=\mu_{1}^{*} \vee \mu_{2}^{+} B^{*-} \\
& \left(\nu \cup \eta^{+}{ }_{B}\right)^{*}{ }_{B}=\mu_{1}^{+*} \vee \mu_{2}^{*} C
\end{aligned}
$$

Type $D$

$$
\begin{gathered}
\eta^{*}{ }^{*}=\mu_{1}^{+} \cup \mu_{2}^{*{ }^{*-}} C^{*} \\
\left(\nu \cup \eta^{*}{ }^{*}\right)^{*}=\mu_{1}^{+*} \vee\left[n_{2}\right]^{*} \vee \mu_{2}^{\prime *-} C^{+} \\
\left(\nu \cup \eta^{*}{ }^{*}\right)^{*}{ }_{D}=\mu_{1}^{+*} \vee \mu_{2}^{-*}{ }_{C}
\end{gathered}
$$

In type $C$, it turns out to be more convenient not to work with $\mu_{2}^{\prime}$. In type $D$, we need to make use of the identity $\eta^{*} D^{*}=\eta^{+-}{ }_{C}$. With these points in mind, the proofs are straightforward.

\section{Proofs of the main theorems in the Classical groups}

In this section, we establish the main theorems of the paper for the classical groups. Theorem 1 is relatively easy: we prove it first, and we make use of it from time to time as we go about proving Theorem 2 . The proof of the latter is broken up into a number of steps and occupies most of the section. The steps may look familiar: we end up proving that $\bar{d}$ has many of the properties established in Section 2 before we show that it is actually an extended duality map.

5.1. The partial order in the classical groups. The strategy for the proof of the theorem below is quite simple: we just attempt the raw computation of the two values of $d_{\mathrm{S}}$, using the techniques from the previous section. Those techniques make it straightforward to find a difference in the answers, starting with a difference in the original marking partitions.

Theorem 5.1. Let $C, C^{\prime} \subset A(\mathcal{O})$ be two conjugacy classes associated to the same orbit. Then, in the classical groups, $d_{\mathrm{S}}(\mathcal{O}, C)=d_{\mathrm{S}}\left(\mathcal{O}, C^{\prime}\right)$ if and only if $C$ and $C^{\prime}$ have the same image in $\bar{A}(\mathcal{O})$. As a consequence, the partial order (1) is well-defined.

Proof. We need to prove that if $C$ and $C^{\prime}$ are two different conjugacy classes in $\bar{A}(\mathcal{O})$, then $d_{\mathrm{S}}(\mathcal{O}, C) \neq$ $d_{\mathrm{S}}\left(\mathcal{O}, C^{\prime}\right)$. Suppose that these conjugacy classes are labelled by ${ }^{\langle\nu\rangle} \lambda$ and ${ }^{\left\langle\nu^{\prime}\right\rangle} \lambda$, respectively. Let $a$ be the largest part of $\lambda$ that appears in only one of $\nu$ and $\nu^{\prime}$. Therefore, $a$ has (generalized) heights of opposite parity in $\nu$ and $\nu^{\prime}$; assume it has even height in $\nu$. That means that we can break ${ }^{\langle\nu\rangle} \lambda$ up into blocks ${ }^{\left\langle\nu_{1}\right\rangle} \lambda_{1} \cup{ }^{\left\langle\nu_{2}\right\rangle} \lambda_{2}$, where the smallest part of $\lambda_{1}$ is $a$. (Note that because $a$ is markable, this is a legitimate division into blocks in whatever type we are working in.) But in ${ }^{\left\langle\nu^{\prime}\right\rangle} \lambda$, there is some basic block ${ }^{\langle\omega\rangle} \zeta, \omega=\left[w_{2}>w_{1}\right]$, such that $w_{2} \geq a>w_{1}$. We build a division into blocks around this basic block, writing ${ }^{\left\langle\nu^{\prime}\right\rangle} \lambda=\left\langle\nu_{1}^{\prime}\right\rangle \lambda_{1}^{\prime} \cup^{\langle\omega\rangle} \zeta \cup^{\left\langle\nu_{2}^{\prime}\right\rangle} \lambda_{2}^{\prime}$. Finally, let $h=\operatorname{ht}_{\lambda}(a)$. 
Let $\mu=d_{\mathrm{S}}\left({ }^{\langle\nu\rangle} \lambda\right)$, and $\mu^{\prime}=d_{\mathrm{S}}\left({ }^{\left\langle\nu^{\prime}\right\rangle} \lambda\right)$. Using Proposition 4.9 just to compute $d_{\mathrm{S}}$, we have

$$
\mu^{*}=d_{\mathrm{S}}\left({ }^{\left\langle\nu_{1}\right\rangle} \lambda_{1}\right)^{*} \cup d_{\mathrm{S}}\left({ }^{\left\langle\nu_{2}\right\rangle} \lambda_{2}\right)^{*} .
$$

We see that $\sigma_{h}\left(\mu^{*}\right)=\left|d_{\mathrm{S}}\left({ }^{\left\langle\nu_{1}\right\rangle} \lambda_{1}\right)^{*}\right|=\left|\lambda_{1}\right|=\sigma_{h}(\lambda)$ in types $C$ or $D$, and $\sigma_{h}\left(\mu^{*}\right)=\left|\lambda_{1}\right|-1=\sigma_{h}(\lambda)-1$ in type $B$. (This comes from just counting the "+" and "- operations that are done in computing $d_{\mathrm{S}}$ in each type.)

We now analyze $\mu^{\prime}$. Write $\zeta=\zeta^{\prime} \cup \zeta^{\prime \prime}$, where $\zeta^{\prime}=\chi_{\mathrm{ht}(a)}^{+}(\zeta)$ and $\zeta^{\prime \prime}=\chi_{\mathrm{ht}(a)}^{-}(\zeta)$. Suppose we are working in type $B$; and suppose further that $\left\langle\nu_{1}^{\prime}\right\rangle \lambda_{1}^{\prime}$ is nontrivial, so that ${ }^{\langle\omega\rangle} \zeta$ is of type $D$. (Definition 4.8 says basic $B$-blocks can only occur at the beginning of a marked $B$-partition.) Then Proposition 4.12 says $d_{\mathrm{S}}\left({ }^{\langle\omega\rangle} \zeta\right)=\zeta^{+-*}{ }_{D}$. We have $\zeta^{+-*}=\zeta^{\prime+*} \vee \zeta^{\prime \prime-*}$, so by Lemma $3.1, \zeta^{+-*}{ }_{D}=\zeta^{\prime+*+}{ }_{D-} \vee \zeta^{\prime \prime-*}{ }_{B}$, so

$$
\mu^{\prime *}=d_{\mathrm{S}}\left({ }^{\left\langle\nu_{1}^{\prime}\right\rangle} \lambda_{1}^{\prime}\right) \cup \zeta^{\prime+*+}{ }_{D-} \cup \zeta^{\prime \prime-*}{ }_{B} \cup \bar{d}\left({ }^{\left\langle\nu_{2}^{\prime}\right\rangle} \lambda_{2}^{\prime}\right) .
$$

We see that $\sigma_{h}\left(\mu^{\prime *}\right)=\left|d_{\mathrm{S}}\left(\left\langle\nu_{1}^{\prime}\right\rangle \lambda_{1}^{\prime}\right)\right|+\left|\zeta^{\prime+*+}{ }_{D-}\right|=\left(\left|\lambda_{1}^{\prime}\right|-1\right)+\left(\left|\zeta^{\prime}\right|+1\right)=\left|\lambda_{1}^{\prime}\right|+\left|\zeta^{\prime}\right|=\sigma_{h}(\lambda)$. A nearly identical argument establishes that $\sigma_{h}\left(\mu^{\prime *}\right)=\sigma_{h}(\lambda)$ when $\left\langle\nu_{1}^{\prime}\right\rangle \lambda_{1}^{\prime}$ is trivial and $\langle\omega\rangle \zeta$ is of type $B$.

Similar computations show that in types $C$ and $D$, we get $\sigma_{h}\left(\mu^{\prime *}\right)=\sigma_{h}(\lambda)+1$. Thus, in every case, we get $\sigma_{h}\left(\mu^{\prime *}\right)=\sigma_{h}\left(\mu^{*}\right)+1$, so $\mu \neq \mu^{\prime}$, as desired.

5.2. Special marked partitions. The remainder of the section is devoted to establishing Theorem 2 for the classical groups. We begin our attack on it by attempting to characterize the marked partitions that occur in the image of $\bar{d}$. In this subsection, we define the set $\tilde{\mathcal{P}}_{X}^{\text {sp }}(n)$ of special marked partitions, and show that the image of $\bar{d}$ is contained with this set. Of course, we still have to prove various properties of $\bar{d}$ before we can know that this terminology coincides with the idea of "special" that we introduced in Section 2.

Definition 5.2. Let ${ }^{\langle\nu\rangle} \lambda$ be a reduced marked partition, with $\nu=\left[n_{l}>\cdots>n_{1}\right]$. Assume that $l$ is even, if necessary by taking $n_{1}=0$ in type $C$. In type $B$ (resp. $C, D$ ), ${ }^{\langle\nu\rangle} \lambda$ is called special if there are no even (resp. odd, even) parts of odd (resp. even, even) height between $n_{2 i}$ and $n_{2 i-1}$ for $i=1, \ldots, l / 2$; that is, if there are no even (resp. odd, even) parts of odd (resp. even, even) height whose (generalized) height in $\nu$ is odd. The set of special marked partitions in $\tilde{\mathcal{P}}_{X}(n)$ is denoted $\tilde{\mathcal{P}}_{X}^{\mathrm{sp}}(n)$.

Note that any trivially marked partition is special by this definition, as we expect from Proposition 2.7. On the other hand, if $\lambda$ is a special partition, a nontrivially marked partition ${ }^{\langle\nu\rangle} \lambda$ may be either special or nonspecial.

Lemma 5.3. We have that $\bar{d}\left({ }^{\langle\varnothing\rangle} \lambda\right)$ is a special marked partition for any $\lambda$.

Proof. Let us consider the situation in type $B$. Recalling Lemma 3.3, we can write $d_{\mathrm{S}}\left({ }^{\langle\varnothing\rangle} \lambda\right)=d_{\mathrm{BV}}(\lambda)=\lambda^{*-}{ }_{C}$. Let $\pi$ be the list of even parts with odd multiplicity in $\lambda^{*}$. In $\lambda^{*-}{ }_{C}$, some parts of $\pi$ have odd multiplicity, and others have even multiplicity. According to Lemma 3.2, we could replace $\pi$ by the set $\pi^{\prime}$ obtained by taking the complementary set of even parts with odd multiplicity, together with the same set of even parts with even multiplicity as $\pi$. That is, $\pi^{\prime}$ is the list of even parts that have even multiplicity in $\lambda^{*-}{ }_{C}$ and odd multiplicity in $\lambda^{*}$, or odd multiplicity in $\lambda^{*-}{ }_{C}$ and even multiplicity in $\lambda^{*}$. We have $d_{\mathrm{S}}(\langle\nu\rangle)=\left\langle\pi{ }^{\langle\pi\rangle} \lambda^{*-}{ }_{C}=\left\langle\pi^{\prime}\right\rangle \lambda^{*-}{ }_{C}\right.$; we work with $\pi^{\prime}$ for the rest of this proof.

What happens as we pass from $\lambda^{*-}$ to $\lambda^{*-}{ }_{C}$ ? We have to make a change in the partition every time we encounter an odd part with odd multiplicity. There are an even number of odd parts with odd multiplicity; we consider them in pairs. Indeed, suppose

$$
a_{1}^{k_{1}}, a_{2}^{k_{2}}, \ldots, a_{l}^{k_{l}}
$$

is a list of consecutive odd parts of $\lambda^{*-}$, with $a_{1}$ and $a_{l}$ being, say, the largest two odd parts with odd multiplicity. (We are not requiring that $a_{i}$ and $a_{i+1}$ be consecutive in $\lambda^{*-}$, but merely that any parts between them be even.) We have assumed that the multiplicities $k_{1}$ and $k_{l}$ are odd, while $k_{2}, \ldots, k_{l-1}$ are even. Then, the $C$-collapse replaces the above parts by the following ones:

$$
a_{1}^{k_{1}-1}, a_{1}-1, a_{2}+1, a_{2}^{k_{2}-2}, a_{2}-1, \ldots, a_{l}+1, a_{l}^{k_{l}-1} .
$$

On each such pair of odd parts with odd multiplicity, the $C$-collapse follows the pattern of the change from (17) to (18); we just investigate what happens on one instance of the pattern. Listing the even parts 
that have changed multiplicities, we obtain

$$
\pi^{\prime}=\left[a_{1}-1, a_{2}+1, a_{2}-1, \ldots, a_{l}+1\right] .
$$

Note that because $\lambda^{*-}$ has no odd parts between $a_{i-1}$ and $a_{i}$, there are no odd parts between $a_{i-1}-1$ and $a_{i}+1$ in $\lambda^{*-} C$.

Now, the key observation here is that $\lambda^{*-} C$ is a special $C$-partition (it equals $d_{\mathrm{BV}}(\lambda)$ ). Odd parts in special $C$-partitions have even height and even multiplicity, so any part immediately greater than an odd part also has even height. In particular, each $a_{i}+1$ has even height, and is therefore markable. When we pass to the reduced label, for each $i$, we either retain both parts $a_{i-1}-1$ and $a_{i}+1$ (if $a_{i-1}-1$ has even height), or eliminate both of them (if $a_{i-1}-1$ has odd height). Since there are no odd parts between these two parts for any $i$, we have a special marked partition.

Proposition 5.4. We have that $\bar{d}\left({ }^{\langle\nu\rangle} \lambda\right)$ is a special marked partition for any ${ }^{\langle\nu\rangle} \lambda$.

Proof. The previous lemma establishes this fact for trivially marked blocks, so now we need only consider basic blocks. This is easy to deduce from the formulas given in Proposition 4.12; we work it out in type $B$ now. Let ${ }^{\langle\nu\rangle} \lambda$ be a type- $B$ basic block with $\nu=\left[n_{2}>n_{1}\right]$, and let $h_{1}=\operatorname{ht}_{\lambda}\left(n_{1}\right)$ : we have that $h_{1}$ is odd. We can write $\lambda=\left[1^{h_{1}}\right] \vee \lambda^{\prime}$, where $\lambda^{\prime}$ is a $C$-partition (since $\lambda$ is a $B$-partition). Then, $\lambda^{-*}{ }_{C}=\left(\left[h_{1}-1\right] \cup \lambda^{* *}\right)_{C}$. (Note that $\left[h_{1}-1\right]$ is probably not superior to $\lambda^{\prime *}$.) Now, $h_{1}-1$ is even, so it is unaffected by the $C$-collapse: $\lambda^{-*}{ }_{C}=\left[h_{1}-1\right] \cup \lambda^{\prime *}{ }_{C}$. Since $\lambda^{\prime}$ is a $C$-partition, $\lambda^{\prime *}{ }_{C}$ is a special $C$-partition, in which all odd parts have even height. We claim that the part $\left[h_{1}-1\right]$ "pushes them down" so that they have odd height. Indeed, the only part of of $\lambda^{\prime *}{ }_{C}$ larger than $h_{1}-1$ is $h_{1}$. That is, to be sure, an odd part with even height (which is equal to its multiplicity, $n_{2}-1$ ), but all other parts of $\lambda^{\prime *}{ }_{C}$ have their heights increased by 1 when we pass to $\lambda^{-*}{ }_{C}$. But since $h_{1}$ is the largest part of $\lambda^{-*}{ }_{C}$, it obviously cannot have odd generalized height in the marking partition. All other odd parts have odd height, so $\bar{d}\left({ }^{\langle\nu\rangle} \lambda\right)$ is special.

5.3. Involutivity. Next, we undertake the task of showing that $\bar{d}$ is an involution on the set of special marked partitions. We do this in several stages, beginning just with the trivial conjugacy class on special orbits, then working up to the trivial conjugacy class for all orbits, and finally to the full special set.

Lemma 5.5. If $\lambda$ is a special partition, then $\bar{d}(\langle\varnothing\rangle\rangle)=\langle\varnothing\rangle_{\mathrm{BV}}(\lambda)$.

Proof. If $\lambda$ is a special $B$ - (resp. $C$-, $D$-) partition, then $\lambda^{*}$ is a $B$ - (resp. $C$-, $C$-) partition, so all its even (resp. odd, odd) parts have even multiplicity. Therefore, the partition $\pi$ defined in (8) is trivial. It follows that $\rho$ in (9) is trivial as well, as is the marking partition of $\bar{d}(\langle\nu\rangle)$. The underlying partition is then given by $d_{\mathrm{S}}\left({ }^{\langle\nu\rangle} \lambda\right)=d_{\mathrm{BV}}(\lambda)$.

Lemma 5.6. For any partition $\lambda$, we have that $\bar{d}^{2}\left({ }^{\langle\varnothing\rangle} \lambda\right)=\langle\varnothing\rangle$.

Proof. We know that $\bar{d}\left({ }^{\langle\varnothing\rangle} \lambda\right)$ is Sommers' canonical inverse for $\lambda$, so that $d_{\mathrm{S}}\left(\bar{d}\left({ }^{\langle\varnothing\rangle} \lambda\right)\right)=\lambda$. We therefore have $\bar{d}^{2}(\langle\varnothing\rangle \lambda)=\langle\nu\rangle \lambda$ for some marking partition $\nu$. We only need to show that $\nu=\varnothing$. To do this, we use Proposition 4.9 to decompose $\lambda$ into pieces as simple as possible. Call a partition of the form $\left[a^{l}\right]$, with $l$ even, a rectangle, and call a partition of the form

$$
\left[a_{1}^{k_{1}}, a_{2}^{k_{2}}, \ldots, a_{m}^{k_{m}}\right]
$$

with $k_{1}$ and $k_{m}$ odd and $k_{2}, \ldots, k_{m-1}$ even, a staircase. Any $D$-partition can be written as a union of rectangles and staircases. Let us define a partial staircase to be a staircase from which either $a_{1}^{k_{1}}$ or $a_{m}^{k_{m}}$ is omitted: a lower partial staircase in the former case, and an upper partial staircase in the latter. In seeking a division into blocks, we can write any $B$-partition as a lower partial staircase followed by some number of rectangles and staircases, and any $C$-partition as a union of rectangles and staircases, possibly followed by an upper partial staircase. The proof of this lemma is accomplished by proving it separately for each of these kinds of blocks.

In many cases, showing that $\nu$ is trivial is easy because $\lambda$ just does not have any possible marking partitions. Rectangles and staircases have only one part of even height, while upper partial staircases have none; and lower partial staircases have only one part of odd height. The lemma follows in completely when $\bar{d}\left({ }^{\langle\varnothing\rangle} \lambda\right)$ is of type $B$ or $D$ (where marking partitions must have an even number of parts), and for upper partial staircases in type $C$. For rectangles in type $C$, the statement is a consequence of Lemma 4.4 . The only remaining case is that of staircases in type $C$, which we treat now. 
Let $\lambda$ be a $C$-partition of the form (20), and let $\mu=d_{\mathrm{S}}\left({ }^{\langle\varnothing\rangle} \lambda\right)$. We have $\mu=\lambda^{+}{ }_{B}{ }^{*}$; moreover, we claim that $\bar{d}\left({ }^{\langle\nu\rangle} \lambda\right)=\langle\varnothing\rangle$. Note that in $\lambda, a_{1}$ and $a_{m}$ must be even. All the parts $a_{1}, \ldots, a_{m-1}$ have odd height; only $a_{m}$ has even height. Therefore, $\lambda^{*}$ has only one even part, its largest one, and the multiplicity of that part is $a_{m}$, which is even. Therefore, $\pi$ as defined in (8) is trivial, so $\vec{d}(\langle\nu\rangle \lambda)$ is trivially marked.

We could just trudge ahead and compute $\bar{d}\left({ }^{\langle\varnothing\rangle} \mu\right)$ directly, but instead, we use the following trick. We have already observed that the proposition holds for $B$-partitions, so $\bar{d}^{2}\left({ }^{\langle\varnothing\rangle} \mu\right)=\left\langle{ }^{\varnothing \varnothing} \mu\right.$. That is, $\bar{d}\left({ }^{\langle\nu\rangle} \lambda\right)=\langle\varnothing\rangle$. But we also have $\bar{d}\left({ }^{\langle\varnothing\rangle} \lambda\right)=\langle\varnothing\rangle \mu$, so if we had $\nu \neq \varnothing$, that would contradict Theorem 5.1. Therefore, $\nu=\varnothing$, and the proposition holds for $C$-staircases.

Lemma 5.7. Let ${ }^{\langle\nu\rangle} \lambda$ be a special ultrabasic block, with $\nu=\left[n_{2}>n_{1}\right]$. If ${ }^{\langle\nu\rangle} \lambda$ is of type B (resp. $C$, D), let $m$ be the largest part of even (resp. odd, even) height in $d_{\mathrm{S}}\left({ }^{\langle\nu\rangle} \lambda\right)$. (When ${ }^{\langle\nu\rangle} \lambda$ is of type B, we put $m=0$ if $d_{\mathrm{S}}\left({ }^{\langle\nu\rangle} \lambda\right)$ has no parts of even height.) If $m>1$, then $\bar{d}\left({ }^{\langle\nu\rangle} \lambda\right)$ is again a special ultrabasic block, given by the following formulas:

$$
\begin{array}{ll}
\text { Type B: } & \bar{d}\left({ }^{\langle\nu\rangle} \lambda\right)=\langle[m]\rangle \lambda^{-*}, \\
\text { Type C: } & \bar{d}\left({ }^{\langle\nu\rangle} \lambda\right)=\langle[m, 1]\rangle \lambda^{+*}, \\
\text { Type D: } & \bar{d}\left({ }^{\langle\nu\rangle} \lambda\right)=\langle[m, 1]\rangle \lambda^{+-*} .
\end{array}
$$

If $m \leq 1$, then $\bar{d}\left({ }^{\langle\nu\rangle} \lambda\right)$ is a trivially marked partition, whose underlying partition is as given above.

Proof. To prove this, we must dive into the details of the proof of Proposition 5.4. Suppose ${ }^{\langle\nu\rangle} \lambda$ is of type $B$; recall that we wrote $\lambda=\left[1^{h_{1}}\right] \vee \lambda^{\prime}$, where $\lambda^{\prime}$ is a $C$-partition. If $\langle\nu\rangle \lambda$ is special, then all its even parts must have even heights. (This is true for even parts smaller than $n_{2}$ by the definition of "special," and for even parts larger than $n_{2}$ by the definition of "basic block.") This means that in $\lambda^{\prime}$, all odd parts have even heights; i.e., $\lambda^{\prime}$ is a special $C$-partition. Therefore, $\lambda^{\prime *}{ }_{C}=\lambda^{\prime *}$, and $\lambda^{-*}{ }_{C}=\left[h_{1}-1\right] \cup \lambda^{\prime *}=\lambda^{-*}$. (Here we have used the fact that $n_{1}=1$, so $h_{1}$ does not occur as a part of $\lambda^{\prime *}$.)

Now, $\lambda^{*}=\left[h_{1}\right] \cup \lambda^{\prime *}$ does not have any odd parts with odd multiplicity other than $h_{1}$, because $\lambda^{\prime *}$ is a $C$-partition. We have $\lambda^{*}=\eta^{*+} \vee\left[1^{n_{2}}\right]$, so in $\eta^{*}$, there are no even parts of height less than or equal to $n_{2}$ that have odd multiplicity. Thus $\pi$ as defined in (8) only has parts of height greater than $n_{2}$. Now, back in $\lambda$, all parts larger than $n_{2}$ have even height, so in $\eta^{*}$ or $\lambda^{*}$, all parts whose height is greater than $n_{2}$ must be even. Let $b$ be the largest part of $\lambda^{*}$ that has even height greater than $n_{2}$; in other words, $b$ is the largest markable part smaller than $h_{2}$. (If there are no markable parts smaller than $h_{2}$, take $b=0$.) There is an odd number of even parts that are greater than or equal to $b$ and smaller than $h_{2}$, but an even number of even parts smaller than $b$ and greater than or equal to any smaller markable part. It follows that when we pass to the reduced marked partition to compute ${ }^{\langle\rho\rangle} \tau$ in $(9)$, we get $\rho=[b]$.

Finally, we look at $\bar{d}\left({ }^{\langle\nu\rangle} \lambda\right)=\langle\hat{\nu} \cup[b]\rangle^{-*}$. Since $b$ is the largest markable part smaller than $h_{2}$, it is clear that we can replace $\left[h_{1}-1>h_{2}-1 \geq b\right]$ by $\left[h_{1}-1\right]$ without changing the reduced marked partition to which it is equivalent. Now, let $m$ be the largest part of even height in $\lambda^{-*}$, or, if there are no parts of even height, take $m=0$. If $m \neq 0$, suppose its height is $k$. That means that in $\lambda, k$ is an even part of height $m$. Since ${ }^{\langle\nu\rangle} \lambda$ is special, $k$ must have even height, so $m$ is necessarily even. Therefore, $m$ is markable in $\lambda^{-*}$. In the case of either $m \neq 0$ or $m=0$, then, we see that $\langle[m]\rangle \lambda^{-*}$ is the reduced marked partition equivalent to $\left\langle\left[h_{1}-1\right]\right\rangle \lambda^{-*}$.

Proposition 5.8. If ${ }^{\langle\nu\rangle} \lambda$ is a special marked partition, then $\bar{d}^{2}\left({ }^{\langle\nu\rangle} \lambda\right)=\langle\nu\rangle$.

Proof. Lemma 5.6 established this fact for trivially marked partitions, so now we only need to consider basic blocks. Indeed, we actually restrict ourselves to ultrabasic blocks, since we can then use Lemma 4.4 to pass up to the result for arbitrary basic blocks. Let ${ }^{\langle\nu\rangle} \lambda$ be a special ultrabasic block, and let $\bar{d}\left({ }^{\langle\nu\rangle} \lambda\right)=\langle\xi\rangle \mu$. According to Lemma 5.7, there are two cases to consider: either $\langle\xi\rangle \mu$ is trivially marked, or it is again a special ultrabasic block.

First, suppose it is trivially marked. In each type, we can directly compute $d_{\mathrm{S}}\left({ }^{(\varnothing)} \mu\right)=d_{\mathrm{BV}}(\mu)$ : in type $B$, for example, we have $\mu=\lambda^{-*}$, and $d_{\mathrm{BV}}(\mu)=\mu^{+} B^{*}{ }_{B}=\lambda^{*}{ }_{B}{ }^{*} B$. (To get $\mu^{+}=\lambda^{*}$, we had to use the fact that $n_{1}=1$.) Moreover, according to Lemma 5.7, the fact that $\bar{d}\left({ }^{\langle\nu\rangle} \lambda\right)$ is trivially marked means that $\mu$ has no parts of even height, which in turn means that $\mu^{*}=\lambda^{-}$has no even parts. Again using that $n_{1}=1$, it follows that $\lambda$ has no even parts, and is therefore automatically a special $B$-partition. We deduce that 
$\lambda^{*}{ }^{*}{ }_{B}=\lambda$. Is it possible that $\bar{d}\left({ }^{\langle\varnothing\rangle} \mu\right)=\left\langle\nu^{\prime}\right\rangle$ for some $\nu^{\prime} \neq \nu$ ? Let us again use the trick from the end of the proof of Lemma 5.6. We know from Lemma 5.6 that $\left.\bar{d}^{2}(\langle\varnothing\rangle) \mu\right)={ }^{\langle\varnothing\rangle} \mu$, but having $\left.\bar{d}\left({ }^{\left\langle\nu^{\prime}\right\rangle} \lambda\right)=\langle\varnothing\rangle\right) \mu=\bar{d}\left({ }^{\langle\nu\rangle} \lambda\right)$ for $\nu^{\prime} \neq \nu$ would contradict Theorem 5.1. Thus, $\bar{d}^{2}\left({ }^{\langle\nu\rangle} \lambda\right)=\langle\nu\rangle$.

Now, suppose instead that $\xi \neq \varnothing$. This time, $\langle\xi\rangle \mu$ is itself a special ultrabasic block, so we can use the formulas of Lemma 5.7 twice in a row to establish the result. For instance, starting in type $B$, we have $\mu=\lambda^{-*}$, so $\bar{d}(\langle\xi\rangle \mu)=\langle[p, 1]\rangle \mu^{+*}$, where $p$ is the largest part of odd height in $\mu^{+*}$. But $\mu^{+*}=\lambda$, as argued in the previous paragraph, and $n_{2}$ is the largest part of odd height in $\lambda$. In this case as well, we find that $\bar{d}^{2}(\langle\nu\rangle \lambda)=\langle\nu\rangle$.

5.4. Specialization. The third step is to define a map for passing from a given marked partition to special one that is larger than it in the partial order. After we show that that this coincides with $\bar{d}^{2}$, we will be in a position prove that $\bar{d}$ is a weak extended duality map, i.e., it satisfies axioms (1)-(3). We begin with a map which we call the partial specialization map $s: \tilde{\mathcal{P}}_{\epsilon}(n) \rightarrow \tilde{\mathcal{P}}_{\epsilon}(n)$, defined as follows. If ${ }^{\langle\nu\rangle} \lambda$ is a nonspecial marked partition of type $B$ (resp. $C, D$ ), let $a$ be the smallest even (resp. odd, even) part of odd (resp. even, even) height in $\lambda$ and odd height in $\nu$. (Of course, no such $a$ exists for a special marked partition). The part $a$ must have even multiplicity, say $l$. Let $\lambda^{\prime}$ be the partition gotten from $\lambda$ by deleting all $l$ copies of $a$. We put

$$
s\left({ }^{\langle\nu\rangle} \lambda\right)= \begin{cases}\langle\nu\rangle & \text { if } \\ { }^{\langle\nu\rangle} \lambda \text { is special, } \\ { }^{\langle\nu\rangle}\left(\lambda^{\prime} \cup\left[a+1, a^{l-2}, a-1\right]\right) & \text { if }{ }^{\langle\nu\rangle} \lambda \text { is nonspecial. }\end{cases}
$$

Of course, we may have to pass to the reduced marked partition from the above formula, if it happens that $a+1$ was a markable part of $\lambda$ and was, in fact, marked.

It is clear that for a nonspecial marked partition, the map $s$ decreases the total number of even (resp. odd, even) parts of odd (resp. even, even) height in the underlying partition and odd height in the marking partition. By induction on that quantity, we obtain the following result.

Lemma 5.9. Given a marked partition ${ }^{\langle\nu\rangle} \lambda$, there is some nonnegative integer $N$ such that $s^{N}\left({ }^{\langle\nu\rangle} \lambda\right)$ is special.

We now define the specialization map $e: \tilde{\mathcal{P}}_{\epsilon}(n) \rightarrow \tilde{\mathcal{P}}_{\epsilon}^{\mathrm{sp}}(n)$ as

$$
e\left({ }^{\langle\nu\rangle} \lambda\right)=s^{N}\left({ }^{\langle\nu\rangle} \lambda\right)
$$

where $N$ is taken large enough that the right-hand side is special. Note that since the map $s$ fixes special marked partitions, there is no ambiguity in the above definition arising from the particular choice of $N$.

Proposition 5.10. We have that $\bar{d}\left({ }^{\langle\nu\rangle} \lambda\right)=\bar{d}\left(s\left({ }^{\langle\nu\rangle} \lambda\right)\right)$ for any marked partition ${ }^{\langle\nu\rangle} \lambda$.

Proof. We begin by proving that $d_{\mathrm{S}}\left({ }^{\langle\nu\rangle} \lambda\right)=d_{\mathrm{S}}\left(s\left({ }^{\langle\nu\rangle} \lambda\right)\right)$. Let us assume that ${ }^{\langle\nu\rangle} \lambda$ is a nonspecial basic block of type $B$, let $a$ and $l$ be as in the definition of $s$, and let $h=h_{\lambda}(a)$. Let ${ }^{\langle\omega\rangle} \zeta=s\left({ }^{\langle\nu\rangle} \lambda\right)=(\omega, \kappa)$, and let $b$ be the next smaller part of $\lambda$ after $a$. Since $a$ is the smallest even part with odd height, $b$ must either be odd or have even height. But if $b$ has even height, it must have odd multiplicity (since $a$ has odd height), so $b$ is necessarily odd in all cases. Suppose $\nu=\left[n_{2}>n_{1}\right]$. If $n_{2}>a+1$, then $\omega=\nu$; otherwise, $\omega=\left[m>n_{1}\right]$, where $m$ is the largest odd part of $\lambda$ that is smaller than $a$ and has odd height.

Now, $h$ is an odd part with even height $a$ in $\lambda^{*}$, and $h-l$ (also odd) is the next smaller part after $h$. Write $\lambda^{-*}=\tau_{1} \cup\left[h^{a-b}, h-l\right] \cup \tau_{2}$, where $\tau_{1}=\chi_{b}^{+}\left(\lambda^{-*}\right)$ and $\tau_{2}=\chi_{a-1}^{-}\left(\lambda^{-*}\right)$. Using Lemma 3.1, it is easy to check that

$$
d_{\mathrm{S}}\left({ }^{\langle\nu\rangle} \lambda\right)=\lambda^{-*}{ }_{C}=\tau_{1 C} \cup\left[h^{a-b-1}, h-1, h-l+1\right] \cup \tau_{2 C} .
$$

We get $\zeta$ from $\lambda$ by replacing $\left[a^{l}\right]$ by $\left[a+1, a^{l-2}, a-1\right]$. Then $\zeta^{*}$ looks like $\lambda^{*}$, except that the portion of the form $\left[h^{a-b}, h-l\right]$ has been changed to $\left[h^{a-b-1}, h-1, h-l+1\right]$. In the case that $\omega=\nu$, we just compare with the above computation to see that $\lambda^{-*}{ }_{C}=\zeta^{-*}{ }_{C}$; i.e., $d_{\mathrm{S}}\left({ }^{\langle\nu\rangle} \lambda\right)=d_{\mathrm{S}}(s(\langle\nu\rangle))$. But even if $\omega \neq \nu$, we recall that it is not necessary to pass to reduced marked partition when computing $d_{\mathrm{S}}$ (which is, after all, defined as a map $\mathcal{N}_{\mathrm{o}, \mathrm{c}} \rightarrow{ }^{L} \mathcal{N}_{\mathrm{o}}$ ), so we can simply replace $\omega$ by $\nu$ and apply the above argument anyway.

It remains to verify that $\bar{d}\left({ }^{\langle\nu\rangle} \lambda\right)$ and $\bar{d}\left(s\left({ }^{(\nu\rangle} \lambda\right)\right)$ produce the same marking partition. This is straightforward but extremely tedious. The proof consists of writing down the various intermediate marked partitions occurring in (9), while scrupulously remembering to pass to a reduced marked partition whenever possible. The cases of $\omega=\nu$ and $\omega \neq \nu$ must be considered separately; the former is slightly easier. We omit the details. 
Corollary 5.11. We have that $\bar{d} \circ e=\bar{d}$, and that $\bar{d}^{2}=e$.

Proof. The first statement is an immediate consequence of the preceding proposition, since, by induction, we have $\bar{d}=\bar{d} \circ s^{N}$ for all $N \geq 0$. Then, on the one hand, we can apply $\bar{d}$ to both sides again to obtain $\bar{d}^{2} \circ e=\bar{d}^{2}$; but on the other hand, we know by Proposition 5.8 that $\bar{d}^{2}$ is the identity on special marked partitions, and the image of $e$ consists of special marked partitions, so $\bar{d}^{2} \circ e=e$. Thus $\bar{d}^{2}=e$.

Proposition 5.12. We have that $\bar{d}^{2}\left({ }^{\langle\nu\rangle} \lambda\right) \geq{ }^{\langle\nu\rangle} \lambda$ for any marked partition ${ }^{\langle\nu\rangle} \lambda$.

Proof. It is easy to see, by construction, that the underlying partition of $s\left({ }^{\langle\nu\rangle} \lambda\right)$ dominates $\lambda$. Combining this with Proposition 5.10, we see that $s\left({ }^{\langle\nu\rangle} \lambda\right) \geq{ }^{\langle\nu\rangle} \lambda$. It follows that $e\left({ }^{\langle\nu\rangle} \lambda\right) \geq{ }^{\langle\nu\rangle} \lambda$; i.e. that $\bar{d}^{2}\left({ }^{\langle\nu\rangle} \lambda\right) \geq{ }^{\langle\nu\rangle} \lambda$, as desired.

Lemma 5.13. Suppose that ${ }^{\langle\nu\rangle} \lambda \leq\left\langle\nu^{\prime}\right\rangle \lambda^{\prime}$, and that ${ }^{\left\langle\nu^{\prime}\right\rangle} \lambda^{\prime}$ is special. Then $s\left({ }^{\langle\nu\rangle} \lambda\right) \leq{ }^{\left\langle\nu^{\prime}\right\rangle} \lambda^{\prime}$ as well.

Proof. The argument used to prove this statement is similar in flavor to the argument we gave for Theorem 5.1. Assume that ${ }^{\langle\nu\rangle} \lambda$ is nonspecial. Let $\mu=d_{\mathrm{S}}\left({ }^{\langle\nu\rangle} \lambda\right)$ and $\mu^{\prime}=d_{\mathrm{S}}\left({ }^{\left\langle\nu^{\prime}\right\rangle} \lambda^{\prime}\right)$. We have that $\lambda \leq \lambda^{\prime}$ and $\mu \geq \mu^{\prime}$. Since $d_{\mathrm{S}}\left(s\left({ }^{\langle\nu\rangle} \lambda\right)\right)=d_{\mathrm{S}}\left({ }^{\langle\nu\rangle} \lambda\right)$, all we have to prove is that the underlying partition of $s\left({ }^{\langle\nu\rangle} \lambda\right)$ is smaller than $\lambda^{\prime}$. Let $\zeta$ denote the underlying partition of $s\left({ }^{\langle\nu\rangle} \lambda\right)$. Let $a, l$, and $h$ be as in the proof of Proposition 5.10. A brief consideration of how $\zeta$ is formed reveals the following relationship between $\zeta$ and $\lambda$ :

$$
\begin{aligned}
\sigma_{h-l+i}(\zeta) & =\sigma_{h-l+i}(\lambda)+1 & & \text { for } i=1, \ldots, l-1 \\
\sigma_{k}(\zeta) & =\sigma_{k}(\lambda) & & \text { for } k \neq h-l+1, \ldots, h-1
\end{aligned}
$$

We know $\sigma_{k}(\lambda) \leq \sigma_{k}\left(\lambda^{\prime}\right)$ for all $k$, but to establish $\zeta \leq \lambda^{\prime}$, we need to prove the following stronger statements:

$$
\sigma_{h-l+i}(\lambda)+1 \leq \sigma_{h-l+i}\left(\lambda^{\prime}\right) \quad \text { for } i=1, \ldots, l-1
$$

Let us assume that the above fails for some $i$; we shall derive a contradiction. Suppose, in particular, that it fails for $i=j$. This means that $\sigma_{h-l+j}(\lambda)=\sigma_{h-l+j}\left(\lambda^{\prime}\right)$. Let $b_{1} \geq \cdots \geq b_{l}$ be the $(h-l+1)$-th, $\ldots, h$-th parts of $\lambda^{\prime}$, respectively. We have

$$
\sigma_{h-l+j+1}(\lambda)=\sigma_{h-l+j}(\lambda)+a \leq \sigma_{h-l+j+1}\left(\lambda^{\prime}\right)=\sigma_{h-l+j}\left(\lambda^{\prime}\right)+b_{j+1},
$$

so $a \leq b_{j+1}$. We also have

$$
\sigma_{h-l+j-1}(\lambda)=\sigma_{h-l+j}(\lambda)-a \leq \sigma_{h-l+j-1}\left(\lambda^{\prime}\right)=\sigma_{h-l+j}\left(\lambda^{\prime}\right)-b_{j},
$$

which implies $a \geq b_{j}$. Since $b_{j} \geq b_{j+1} \geq a$, we conclude that $a=b_{j}=b_{j+1}$. But then (22) says that $\sigma_{h-l+j+1}(\lambda)=\sigma_{h-l+j+1}\left(\lambda^{\prime}\right)$, so $(21)$ fails for $i=j+1$ as well. If $i_{0}$ is the smallest value of $i$ for which (21) fails, we see by induction that it fails for $i=i_{0}+1, \ldots, l$ as well. Furthermore, $b_{i_{0}}=\cdots=b_{l}=a$.

We claim, moreover, that $i_{0}=1$; i.e. that (21) fails for all $i$. If not, the inequality (23) can be strengthened using the fact that the $\left(i_{0}-1\right)$-th inequality in $(21)$ holds:

$$
\sigma_{h-l+i_{0}-1}(\lambda)+1=\sigma_{h-l+i_{0}}(\lambda)-a+1 \leq \sigma_{h-l+i_{0}-1}\left(\lambda^{\prime}\right)=\sigma_{h-l+i_{0}}\left(\lambda^{\prime}\right)-b_{i_{0}} .
$$

We deduce that $a-1 \geq b_{i_{0}}$. Since $b_{i_{0}} \geq b_{i_{0}+1} \geq a$, we obtain $a-1 \geq a$, a contradiction. We thus have $\sigma_{h-l+i}(\lambda)=\sigma_{h-l+i}\left(\lambda^{\prime}\right)$ and $b_{i}=a$ for $i=1, \ldots, l$. Additionally, (23) also gives us that $\sigma_{h-l}(\lambda)=\sigma_{h-l}\left(\lambda^{\prime}\right)$.

We claim that $a$ must have odd height in $\lambda^{\prime}$ if we are in type $B$, and even height in types $C$ and $D$. We prove it in type $B$ as follows. The first $h-l$ parts of $\lambda$ constitute a $B$-partition, so $\sigma_{h-l}(\lambda)=\sigma_{h-l}\left(\lambda^{\prime}\right)$ is odd. Suppose $a$ had even height in $\lambda^{\prime}$, and let $k$ be the height of the next larger part of $\lambda^{\prime}$. We know that $k$ must be even too, since $a$ must have even multiplicity. Then $\sigma_{h-l}\left(\lambda^{\prime}\right)=\sigma_{k}\left(\lambda^{\prime}\right)+(h-l-k) a$. Since the second term here is even, $\sigma_{k}\left(\lambda^{\prime}\right)$ must be odd. But since $k$ is even, the first $k$ parts of $\lambda^{\prime}$ constitute a $D$-partition, and $\sigma_{k}\left(\lambda^{\prime}\right)$ has to be even. We have a contradiction; therefore, $a$ has odd height in $\lambda^{\prime}$.

Since ${ }^{\left\langle\nu^{\prime}\right\rangle} \lambda^{\prime}$ is special, a must have even height with respect to $\nu^{\prime}$; it cannot appear inside a basic block. We continue to take $k$ to be the height of the next larger part of $\lambda^{\prime}$ after $a$, but we know now that $k$ is odd. Let $\theta^{\prime}=\chi_{k}^{+}\left(\lambda^{\prime}\right)$, and let $\kappa^{\prime}$ be the partition consisting of those parts of $\nu^{\prime}$ that are larger than $a$. Then $\left\langle\kappa^{\prime}\right\rangle \theta^{\prime}$ is a marked $B$-partition, and if we let $m=r_{\lambda^{\prime}}(a)$, then the expression ${ }^{\left\langle\kappa^{\prime}\right\rangle} \theta^{\prime} \cup^{\langle\varnothing\rangle}\left[a^{m}\right] \cup \cdots$ is part of a division into blocks of $\left\langle\nu^{\prime}\right\rangle \lambda^{\prime}$. We can compute, then, that

$$
\mu^{\prime *}=d_{\mathrm{S}}\left(\left\langle\kappa^{\prime}\right\rangle \theta^{\prime}\right)^{*} \cup\left[a^{m}\right] \cup \cdots .
$$


Since $\left\langle\kappa^{\prime}\right\rangle \theta^{\prime}$ is of type $B$, we compute that

$$
\sigma_{h-l}\left(\mu^{\prime *}\right)=\left|\theta^{\prime}\right|-1+(h-l-k) a=\sigma_{h-l}\left(\lambda^{\prime}\right)-1 .
$$

On the other hand, in ${ }^{\langle\nu\rangle} \lambda$, the part a belongs to some nonspecial basic block ${ }^{\langle\gamma\rangle} \phi$, around which we can build a division into blocks ${ }^{\langle\kappa\rangle} \theta \cup{ }^{\langle\gamma\rangle} \phi \cup \ldots$. We either have that ${ }^{\langle\kappa\rangle} \theta$ is of type $B$ and ${ }^{\langle\kappa\rangle} \phi$ of type $D$, or that ${ }^{\langle\kappa\rangle} \theta$ is trivial and ${ }^{\langle\kappa\rangle} \phi$ is of type $B$. Assume we are in the former case; the latter is handled similarly. Let $h^{\prime}=\mathrm{ht}_{\phi}(a)$, and let $\phi^{\prime}=\chi_{h^{\prime}-l}^{+}(\phi)$ and $\phi^{\prime \prime}=\chi_{h^{\prime}-l}^{-}(\phi)$. Using Proposition 4.12 and Lemma 3.1 to compute $d_{\mathrm{S}}(\langle\gamma\rangle \phi)$, we fine

$$
\mu^{*}=d_{\mathrm{S}}(\langle\kappa\rangle \theta)^{*} \cup \phi^{+*++}{ }_{D-}{ }^{*} \cup \phi^{\prime \prime-*}{ }_{B}{ }^{*} \cup \cdots .
$$

We obtain

$$
\sigma_{h-l}\left(\mu^{*}\right)=\left(\left|\theta^{\prime}\right|-1\right)+\left(\left|\phi^{\prime}\right|+1\right)=\sigma_{h-l}(\lambda)
$$

In particular, we see that $\sigma_{h-l}\left(\mu^{*}\right) \not \leq \sigma_{h-l}\left(\mu^{\prime *}\right)$, which contradicts the assumption that $\mu^{*} \leq \mu^{\prime *}$. Therefore, the inequalities (21) hold for all $i$, and we obtain $\zeta \leq \lambda^{\prime}$, as desired.

Proposition 5.14. If ${ }^{\langle\nu\rangle} \lambda \leq{ }^{\left\langle\nu^{\prime}\right\rangle} \lambda^{\prime}$, then $\bar{d}\left({ }^{\langle\nu\rangle} \lambda\right) \geq \bar{d}\left({ }^{\left\langle\nu^{\prime}\right\rangle} \lambda^{\prime}\right)$.

Proof. We first prove the statement in the special case that ${ }^{\langle\nu\rangle} \lambda$ and $\left\langle\nu^{\prime}\right\rangle \lambda^{\prime}$ are special. The $\mathcal{N}_{\mathrm{o}, \overline{\mathrm{c}}}$-inequality $\bar{d}(\langle\nu\rangle \lambda) \geq \bar{d}\left(\left\langle\nu^{\prime}\right\rangle \lambda^{\prime}\right)$ is equivalent to the two $\mathcal{N}_{\mathrm{o}}$-inequalities

$$
d_{\mathrm{S}}\left({ }^{\langle\nu\rangle} \lambda\right) \geq d_{\mathrm{S}}\left({ }^{\left\langle\nu^{\prime}\right\rangle} \lambda^{\prime}\right) \quad \text { and } \quad d_{\mathrm{S}}\left(\bar{d}\left({ }^{\langle\nu\rangle} \lambda\right)\right) \leq d_{\mathrm{S}}\left(\bar{d}\left({ }^{\left\langle\nu^{\prime}\right\rangle} \lambda^{\prime}\right)\right)
$$

The first of these is implied by ${ }^{\langle\nu\rangle} \lambda \leq{ }^{\left\langle\nu^{\prime}\right\rangle} \lambda^{\prime}$, by definition. For the second, since these marked partitions are special, we know $d_{\mathrm{S}}\left(\bar{d}\left({ }^{\langle\nu\rangle} \lambda\right)\right)=\lambda$ and $d_{\mathrm{S}}\left(\bar{d}\left({ }^{\left\langle\nu^{\prime}\right\rangle} \lambda^{\prime}\right)\right)=\lambda^{\prime}$. But the inequality $\lambda \leq \lambda^{\prime}$ is again part of the definition of $\langle\nu\rangle, \lambda \leq\left\langle\nu^{\prime}\right\rangle \lambda^{\prime}$. Thus, (25) holds, and the proposition holds for special marked partitions.

Now, if ${ }^{\langle\nu\rangle} \lambda$ and $\left\langle\nu^{\prime}\right\rangle \lambda^{\prime}$ are arbitrary marked partitions with ${ }^{\langle\nu\rangle} \lambda \leq\left\langle\nu^{\prime}\right\rangle \lambda^{\prime}$, we obtain $\left.{ }^{\langle\nu\rangle} \lambda \leq \bar{d}^{2}\left({ }^{\prime}\right\rangle \lambda^{\prime}\right)$ by Proposition 5.12. Then, repeated application of Lemma 5.13 implies that $e\left({ }^{\langle\nu\rangle} \lambda\right)=\bar{d}^{2}\left({ }^{\langle\nu\rangle} \lambda\right) \leq \bar{d}^{2}\left(\left\langle\nu^{\prime}\right\rangle \lambda^{\prime}\right)$. Both sides of this inequality are special marked partitions, so the previous paragraph tells us that $\bar{d}^{3}\left({ }^{\langle\nu\rangle} \lambda\right) \geq$ $\bar{d}^{3}\left({ }^{\left\langle\nu^{\prime}\right\rangle} \lambda^{\prime}\right)$. Finally, Proposition 5.8, combined with Proposition 5.4, says that $\bar{d}^{3}=\bar{d}$, so we get $\bar{d}\left({ }^{\langle\nu\rangle} \lambda\right) \geq$ $\bar{d}(\langle\nu\rangle \lambda)$, as desired.

5.5. Maximality of the image. We have now established that $\bar{d}$ satisfies each of the axioms (1), (2), and (3), in Propositions 5.14, 5.12, and 4.2, respectively. Only axiom (4) remains, but we are not going to verify it directly. Instead, we employ the strategy developed in Section 2: we need only prove that the set of special marked partitions corresponds to the set $\mathcal{N}_{\mathrm{o}, \overline{\mathrm{c}}}^{\mathrm{sp}}$ defined there, and then the theorem follows by application of Proposition 2.6 .

Lemma 5.15. Let ${ }^{\langle\nu\rangle} \lambda$ be a nonspecial basic block, and let $\mu=d_{\mathrm{S}}\left({ }^{\langle\nu\rangle} \lambda\right)$. There does not exist a marking partition $\xi$ such that $d_{\mathrm{S}}\left({ }^{\langle\xi\rangle} \mu\right)=\lambda$.

Proof. Let us assume that ${ }^{\langle\nu\rangle} \lambda$ is a marked partition of type $B$. Since ${ }^{\langle\nu\rangle} \lambda$ is not special, we can apply the partial specialization map to it and obtain ${ }^{\left\langle\nu^{\prime}\right\rangle} \lambda^{\prime}=s\left({ }^{\langle\nu\rangle} \lambda\right)$, where $\lambda^{\prime}>\lambda$. A brief consideration of the definition of $s$ reveals that ${ }^{\left\langle\nu^{\prime}\right\rangle} \lambda^{\prime}$ must itself be either a basic block or a trivially marked partition; moreover, the latter can be obtained only if $\lambda^{\prime}$ has no parts of odd height except the last one. It is easily verified that for trivially marked partitions with this property, $d_{\mathrm{S}}$ is given by the formulas of Proposition 4.12: thus, $d_{\mathrm{S}}\left(\left\langle\nu^{\prime}\right\rangle \lambda^{\prime}\right)=\lambda^{\prime-*}{ }_{C}$ regardless of whether $\nu^{\prime}$ is trivial or not.

Next, we prove that for any $\xi$,

$$
d_{\mathrm{S}}\left({ }^{\langle\xi\rangle} \mu\right) \geq \mu^{+*}{ }_{B} \geq \lambda^{\prime}
$$

(The appropriate expressions for the middle term in types $C$ and $D$ are $\mu^{-*}{ }_{C}$ and $\mu^{+-*}{ }_{D}$, respectively: these formulas are those appearing in Proposition 4.12.) The lemma then follows, because we will have that $d_{\mathrm{S}}(\langle\xi\rangle \mu)>\lambda$ for all $\xi$.

We shall make the assumption that the smallest part of $\lambda^{\prime}$ is 1 . If $\nu^{\prime}$ is not trivial, we are just assuming that ${ }^{\left\langle\nu^{\prime}\right\rangle} \lambda^{\prime}$ is an ultrabasic block; (26) then follows for general basic blocks by Lemma 4.4, as usual. If $\nu^{\prime}$ is trivial, the same reduction still works, because the fact that only the last part of $\lambda^{\prime}$ has odd height implies that it has odd multiplicity, and is therefore odd. 
For the left-hand inequality of (26), suppose $\langle\xi\rangle \mu=(\xi, \omega)$. Then (note that this is a type- $C$ marked partition) we have

$$
\xi \cup \omega^{+}{ }_{B} \leq \mu^{+}
$$

and thence $\left(\xi \cup \omega^{+}{ }_{B}\right)_{B}^{*} \geq \mu^{+*}{ }_{B}$. For the right-hand inequality, it is easily verified that $\lambda^{\prime-*}{ }_{C}{ }^{+*} \geq \lambda^{\prime}$, although we need to use the fact that the smallest part of $\lambda^{\prime}$ is 1 . It then follows that $\mu^{+*}{ }_{B}=\lambda^{\prime-*}{ }_{C}^{+*}{ }_{B} \geq \lambda_{B}^{\prime}=\lambda^{\prime}$.

Proposition 5.16. Let ${ }^{\langle\nu\rangle} \lambda$ be any nonspecial marked partition, and let $\mu=d_{\mathrm{S}}\left({ }^{\langle\nu\rangle} \lambda\right)$. There does not exist a marking partition $\xi$ such that $\left.d_{\mathrm{S}}(\langle\xi\rangle) \mu\right)=\lambda$.

Proof. We employ induction on the number of parts of $\lambda$. The previous lemma handles the case where ${ }^{\langle\nu\rangle} \lambda$ is a basic block. Otherwise, choose some division into blocks ${ }^{\langle\nu\rangle} \lambda=\left\langle\nu_{1}\right\rangle \lambda_{1} \cup{ }^{\left\langle\nu_{2}\right\rangle} \lambda_{2}$, and let $\mu_{i}=d_{\mathrm{S}}\left({ }^{\left\langle\nu_{i}\right\rangle} \lambda_{i}\right)$ for $i=1$, 2. Suppose we have some $\xi$ such that $d_{\mathrm{S}}\left({ }^{\langle\xi\rangle} \mu\right)=\lambda$. If it is possible to write ${ }^{\langle\xi\rangle} \mu$ as ${ }^{\left\langle\xi_{1}\right\rangle} \mu_{1} \vee\left\langle\xi_{2}\right\rangle \mu_{2}$ for some $\xi_{1}$ and $\xi_{2}$, then it would follow that $d_{\mathrm{S}}\left(\left\langle\xi_{i}\right\rangle \mu_{i}\right)=\lambda_{i}$ for each $i$. But at least one of the $\left\langle\xi_{i}\right\rangle \lambda_{i}$ is nonspecial, so that would contradict the inductive hypothesis.

Suppose, on the other hand, that $\langle\xi\rangle \mu$ cannot be written as such a join. In this case, we again use the technique employed for Theorem 5.1 and Lemma 5.13. Write ${ }^{\left\langle\nu_{1}\right\rangle} \lambda_{1}$ as ${ }^{\langle\varnothing\rangle}\left[a^{l}\right] \vee\left\langle\nu_{1}^{\prime}\right\rangle \lambda_{1}^{\prime}$, where the latter is a decomposition satisfying the hypotheses of Lemma 4.4, chosen such that $a$ is as large as possible. In particular, $a$ will be at least as large as the largest part of $\lambda_{2}$. It is clear that $\sigma_{a}\left(\lambda^{*}\right)=\left|\lambda_{2}\right|+a l$. A brief glance at the formulas of Proposition 4.9 shows that, moreover, $\sigma_{a}(\mu)=\sigma_{a}\left(\lambda^{*}\right)$ if ${ }^{\langle\nu\rangle} \lambda$ is of type $B$ or $D$, but $\sigma_{a}(\mu)=\sigma_{a}\left(\lambda^{*}\right)+1$ in type $C$.

We now turn our attention to ${ }^{\langle\xi\rangle} \mu$. Let $\zeta=d_{\mathrm{S}}\left({ }^{\langle\xi\rangle} \mu\right)$. The assumption that $\langle\xi\rangle \mu$ cannot be written as an appropriate kind of join means that the $a$-th part of $\mu$ has odd (generalized) height in $\xi$. A calculation much like that carried out for the proof of Theorem 5.17, whose details we omit, shows that $\sigma_{a}\left(\zeta^{*}\right)$ is equal to $\sigma_{a}(\mu)$ if $\langle\xi\rangle$ is of type $B$ (i.e., if ${ }^{\langle\nu\rangle} \lambda$ is of type $C$ ), or to $\sigma_{a}(\mu)+1$ if $\langle\xi\rangle \mu$ is of type $C$ or $D$. Thus, in all cases, we have $\sigma_{a}\left(\zeta^{*}\right)=\sigma_{a}\left(\lambda^{*}\right)+1$. In particular, this means that $\zeta \neq \lambda$, contradicting our assumption.

The preceding proposition says exactly that the set of special marked partitions coincides with the set $\mathcal{N}_{\mathrm{o}, \overline{\mathrm{c}}}^{\mathrm{sp}}$ defined in Section 2, as promised. We therefore obtain the following theorem.

Theorem 5.17. The map $\bar{d}$ is the unique extended duality map in the classical groups.

\section{EXPLicit CALCULATIONS AND THE EXCEPTIONAL GROUPS}

The main results in the case of the exceptional groups are established by explicit calculation. In this section, we present explicit calculations of the partial order and the duality map in all of the exceptional groups, as well as in a number of classical groups of small rank.

We name elements $(\mathcal{O}, C) \in \mathcal{N}_{\mathrm{o}, \overline{\mathrm{c}}}$ in the exceptional groups by a pair of symbols $\left(L_{1}, L_{2}\right)$, where $L_{1}$ is the Bala-Carter notation for $\mathcal{O}$, as found in, say, [7], and $L_{2}$ is the label Sommers assigns to $(\mathcal{O}, C)$ in his generalized Bala-Carter theorem [17]. (Of course, we are only writing down $L_{1}$ for our own convenience, since $L_{2}$ alone determines the orbit.) We deviate from this notation when $C$ is the trivial conjugacy class in $\bar{A}(\mathcal{O})$ : in this case, the generalized Bala-Carter label for $(\mathcal{O}, C)$ is the same as the Bala-Carter label for $\mathcal{O}$, but for the sake of brevity, we write $\left(L_{1}, 1\right)$ rather than $\left(L_{1}, L_{1}\right)$.

A further comment about generalized Bala-Carter labels for pairs $(\mathcal{O}, C)$ is in order, because the generalized Bala-Carter theorem is actually a classification of $\mathcal{N}_{\mathrm{o}, \mathrm{c}}$, not of $\mathcal{N}_{\mathrm{o}, \overline{\mathrm{c}}}$. For most orbits in the exceptional groups, we have $A(\mathcal{O})=\bar{A}(\mathcal{O})$, so this distinction does not matter, but in a handful of cases, $\bar{A}(\mathcal{O})$ has fewer conjugacy classes than $A(\mathcal{O})$. This occurs for two orbits in $F_{4}$, two in $E_{7}$, and seven in $E_{8}$. In all but one of these cases, we have $A(\mathcal{O})=S_{2}$ and $\bar{A}(\mathcal{O})=1$; however, for the orbit $E_{8}\left(b_{6}\right)$ in type $E_{8}$, we have $A(\mathcal{O})=S_{3}, \bar{A}(\mathcal{O})=S_{2}$. In all of these cases, the only ambiguity is that two conjugacy classes of $A(\mathcal{O})$ map to the trivial conjugacy class of $\bar{A}(\mathcal{O})$. (In the $E_{8}\left(b_{6}\right)$ example, only one conjugacy class of $S_{3}$ descends to the nontrivial conjugacy class of $S_{2}$.) In each such situation, we simply ignore the nontrivial class of $A(\mathcal{O})$ that maps to the trivial one in $\bar{A}(\mathcal{O})$, and we designate the latter with a label of the form $\left(L_{1}, 1\right)$.

Theorem 6.1. Let $C, C^{\prime} \subset A(\mathcal{O})$ be two conjugacy classes associated to the same orbit. Then, in the exceptional groups, $d_{\mathrm{S}}(\mathcal{O}, C)=d_{\mathrm{S}}\left(\mathcal{O}, C^{\prime}\right)$ if and only if $C$ and $C^{\prime}$ have the same image in $\bar{A}(\mathcal{O})$. As a consequence, the partial order (1) is well-defined in the exceptional groups. 
Proof. Sommers gives tables of all the values of $d_{\mathrm{S}}$ on all pairs $(\mathcal{O}, C) \in \mathcal{N}_{\mathrm{o}, \mathrm{c}}$ for each exceptional group in [18]. We merely read through this table and verify that the above statement is true.

Theorem 6.2. There exists a unique extended duality map in the case of each exceptional group.

Proof. Once we have drawn out the partial-order diagram of $\mathcal{N}_{\mathrm{o}, \overline{\mathrm{c}}}$ for the exceptional groups, we produce the extended duality map by working backwards from the results of Section 2 . Recall, from that section, the definition of $\mathcal{N}_{\mathrm{o}, \overline{\mathrm{c}}}^{\mathrm{sp}}$ : this ought to be the special set for the extended duality map. We verify by inspection in each type that for each pair $(\mathcal{O}, C) \notin \mathcal{N}_{\mathrm{o}, \overline{\mathrm{c}}}^{\mathrm{sp}}$, there is a unique smallest element of $\mathcal{N}_{\mathrm{o}, \overline{\mathrm{c}}}^{\mathrm{sp}}$ that is larger than it. Next, we define the map $\bar{d}$ by referring to Proposition 2.4 and the proof of Proposition 2.5: the latter tells us how to compute $\bar{d}$ on special pairs, while the former does the same for nonspecial pairs. Finally, we tediously verify that the map thus produced does, in fact, satisfy the first three axioms for an extended duality map. The theorem follows by application of Proposition 2.6.

Below, we have drawn out the full Hasse diagram of the partial-order structure on $\mathcal{N}_{\mathrm{o}, \overline{\mathrm{c}}}$ in types $B$ and $C$ up to rank 4, in type $D$ up to rank 5 , and in all the exceptional groups. In these diagrams, most pairs $(\mathcal{O}, C)$ are special. Ones that are not special are indicated by a solid box $\square$. The number of elements in $\mathcal{N}_{\mathrm{o}, \overline{\mathrm{c}}}$ for $G_{2}$ (resp. $F_{4}, E_{6}, E_{7}, E_{8}$ ) is 7 (resp. 24, 25, 58, 106), and the number of special pairs is 7 (resp. 23, $25,55,98)$.

In type $D$ and the exceptional groups, the duality map $\bar{d}$ itself can be visualized as follows: if the nonspecial pairs are deleted from the diagram, the remaining partial-order diagram has a horizontal axis of symmetry. The duality map on special pairs is given by reflection across this axis; then, Proposition 2.4 tells us how to
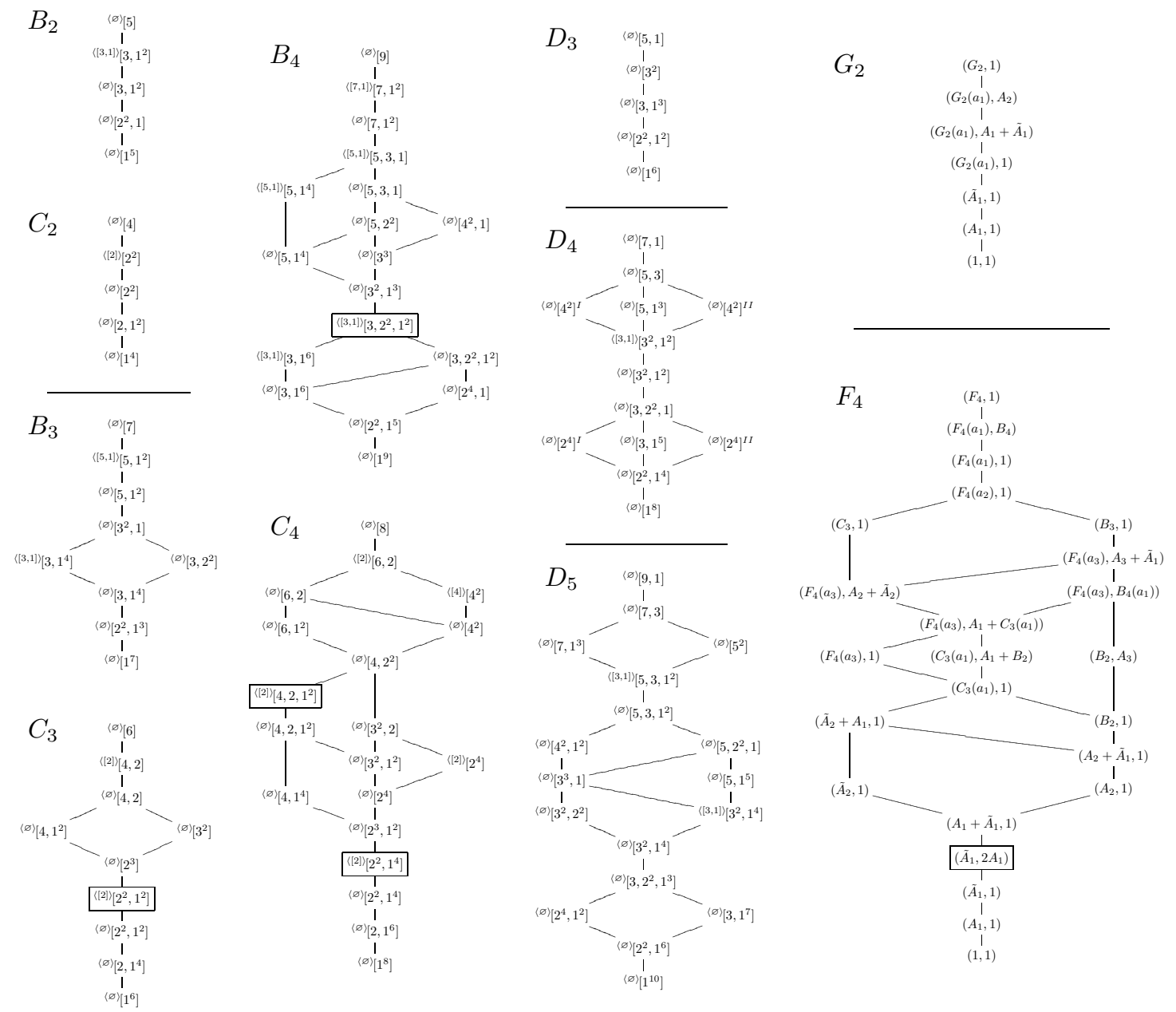

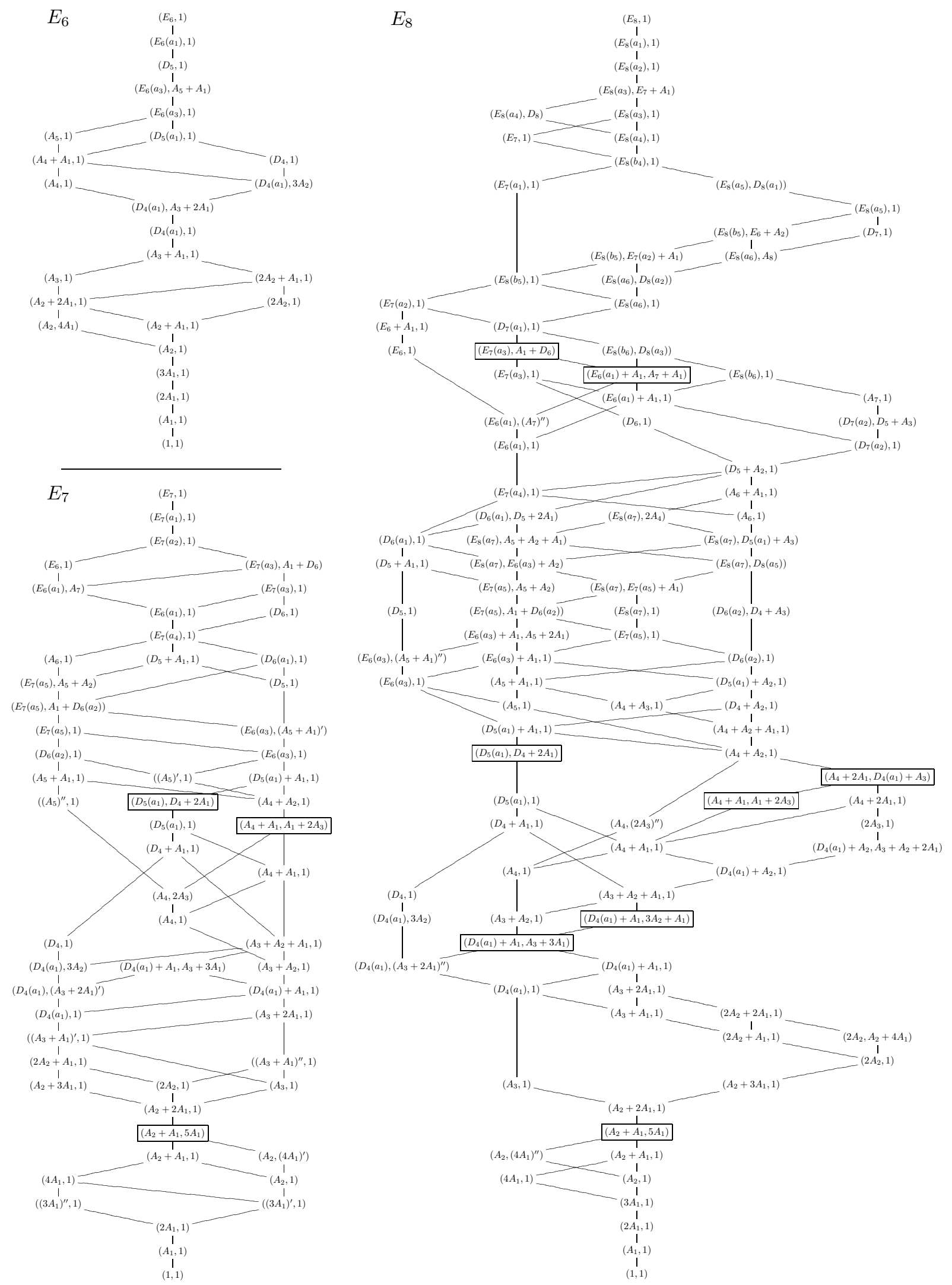
compute $\bar{d}$ on nonspecial pairs. For types $B$ and $C$, we have drawn the Hasse diagram of $\mathcal{N}_{\mathrm{o}, \overline{\mathrm{c}}}\left(B_{n}\right)$ directly above that of $\mathcal{N}_{\mathrm{o}, \overline{\mathrm{c}}}\left(C_{n}\right)$. This combined diagram has a horizontal axis of symmetry if nonspecial pairs are deleted, and $\bar{d}$ is given by reflecting across that.

The observant reader may remark upon an apparent discrepancy between our diagram for $F_{4}$ and those given in other sources (such as [20] or [7]) for the classical duality map. Those sources show that the dual orbit to $B_{3}$ is $\tilde{A}_{2}$, while the dual of $C_{3}$ is $A_{2}$. In our diagram, it looks as though $B_{3}$ and $C_{3}$ have been exchanged. In fact, this discrepancy arises because those sources are illustrating the map $d_{\mathrm{LS}}$, whereas $\bar{d}$ satisfies a compatibility condition with $d_{\mathrm{BV}}$. Remarkably, $d_{\mathrm{LS}}$ and $d_{\mathrm{BV}}$ do not coincide for $F_{4}$, even though it is isomorphic to its Langlands dual. The reason is that passing to the Langlands dual exchanges the long and short roots of the root system, so in corresponding representations of the Weyl groups, the action of the simple reflections corresponding to long and short roots must be interchanged. Alvis [4] describes this corespondence explicitly.

\section{Further COMMENTS}

This final section is devoted to exploring how the partial order and the extended duality map for $\mathcal{N}_{\mathrm{o}, \bar{c}}$ can be employed to enhance understanding and further the study of a handful of topics. I am especially indebted to E. Sommers and A.-M. Aubert for discussions about these matters. Some of the ideas and assertions in this section are the product of joint work with A.-M. Aubert, and will be properly developed and proved in a forthcoming joint paper [2].

In Section 7.1, we show how our new tools can be used to give a uniform approach to existing disparate descriptions of the structure of the groups $\bar{A}(\mathcal{O})$. In Section 7.2, we revisit a conjecture made in [3] regarding the equivariant $K$-theory of the nilpotent cone. We show how to restate it in the language of the partial order on $\mathcal{N}_{\mathrm{o}, \overline{\mathrm{c}}}$, and we then investigate a refinement of the conjecture suggested by the rephrasing. Finally, in Section 7.3, we consider what the partial order might have to say about representations of Weyl groups, via the Springer correspondence.

One issue that we will not address, however, is that of giving an "intrinsic" construction of the duality map. That is, it would be nice to have some representation-theoretic construction explaining why $\bar{d}(\mathcal{O}, C)$ should be associated to $(\mathcal{O}, C)$, rather than merely an opaque existential statement regarding the set $\mathcal{N}_{\mathrm{o}, \overline{\mathrm{c}}}^{\mathrm{sp}}$. Such a construction is likely to elucidate many aspects of the duality map. For instance, what makes a pair $(\mathcal{O}, C)$ special or nonspecial? Is there a way to realize $\mathcal{N}_{\mathrm{o}, \bar{c}}$ geometrically, identifying its elements with certain locally closed subvarieties of some variety in such a way that its partial order just becomes the usual closure order? Finally, is there an analogue of the compatibility that $d_{\mathrm{LS}}$ and $d_{\mathrm{BV}}$ enjoy with induction of nilpotent orbits? An answer to this last question would, of course, require a theory of induction for $\mathcal{N}_{\mathrm{o}, \overline{\mathrm{c}}}$.

7.1. The $\bar{A}(\mathcal{O})$ groups as Coxeter groups. In the exceptional groups, $\bar{A}(\mathcal{O})$ is always just a symmetric group, and therefore a Coxeter group. Indeed, it has a unique Coxeter presentation up to conjugacy. This structure was employed by Lusztig [15] to obtain a correspondence between conjugacy classes of $\bar{A}(\mathcal{O})$ on the one hand, and parabolic subgroups on the other. In the classical groups, however, $\bar{A}(\mathcal{O})$ is a product of many copies of $\mathbb{Z} / 2 \mathbb{Z}$ : regarding this as a $\mathbb{Z} / 2 \mathbb{Z}$-vector space, any basis is a set of simple reflections for a Coxeter presentation. Moreover, no two such presentations are even conjugate. Carrying out an analogue of the constructions in [15] requires choosing a particular Coxeter presentation. This is done for the classical groups in [3] by choosing the simple reflections to be elements of those nontrivial conjugacy classes $C$ for which $d_{\mathrm{S}}(\mathcal{O}, C)$ has maximal dimension. That turned out to be the correct choice for a certain conjecture regarding local systems, which will be discussed in the next section.

The partial order on $\mathcal{N}_{\mathrm{o}, \overline{\mathrm{c}}}$ can be used to give a uniform description of the canonical Coxeter structure of $\bar{A}(\mathcal{O})$ in all types. If we restrict our attention to a single orbit $\mathcal{O}$, then the conjugacy classes of $\bar{A}(\mathcal{O})$ inherit a partial order from $\mathcal{N}_{\mathrm{o}, \overline{\mathrm{c}}}$. The trivial conjugacy class is the smallest element in this partial order, according to Proposition 2.3. Let us call a class $C$ superminimal if it lies just above the trivial class: that is, if $C>C^{\prime}$ implies that $C^{\prime}$ is the trivial class. The choice of simple reflections in [3] consists precisely of elements of superminimal conjugacy classes. The following result will be proved in [2]; it has also been independently obtained by Sommers [19].

Theorem 7.1. There is a set of involutions $S \subset \bar{A}(\mathcal{O})$, unique up to conjugacy, such that:

(a) every element of $S$ is a member of a superminimal conjugacy class, 
(b) every superminimal conjugacy class has at least one representative in $S$, and

(c) $S$ constitutes a set of simple reflections for a presentation of $\bar{A}(\mathcal{O})$ as a Coxeter group.

When studying the representations of Coxeter groups, we have available to us the Macdonald-LusztigSpaltenstein operation of "truncated induction" or " $j$-induction." This operation is defined for a certain class of irreducible representations, which includes all special representations. The truncated induction of an irreducible representation (when it is defined) is the unique irreducible component of the induced representation that occurs in as small a symmetric power of the reflection representation as possible. It turns out that every representation of $\bar{A}(\mathcal{O})$ arises as the truncated induction of the sign representation of some parabolic subgroup, which is uniquely determined up to conjugacy. Parabolic subgroups are, in turn, determined by subsets of the set of simple reflections.

Let $S$ be a set of simple reflections as found by Theorem 7.1, and let $P \subset S$ be a subset. We thus associate a certain representation $\rho_{P}$ of $\bar{A}(\mathcal{O})$ to $P$, and we take $C_{P}$ to be the conjugacy class containing the product of all the elements of $P$. (In [2], for technical reasons, the formula for $\rho_{P}$ is not simply the truncated induction of the sign representation, but rather that tensored with the sign representation of $\bar{A}(\mathcal{O})$.) The following proposition, relating conjugacy classes and representations of $\bar{A}(\mathcal{O})$, collects and rephrases facts that are implicit in the work of Lusztig [15] for the exceptional groups, and in [3] for the classical groups.

Proposition 7.2. $C_{P}$ is well-defined, i.e., independent of the order in which the elements of $P$ are written. Moreover, every conjugacy class of $\bar{A}(\mathcal{O})$ occurs as some $C_{P}$, where $P$ is uniquely determined up to conjugacy. Therefore, the map

$$
\rho_{P} \leadsto C_{P}
$$

is a natural bijection between irreducible representations and conjugacy classes of $\bar{A}(\mathcal{O})$. In addition, we have that $\left(\mathcal{O}, C_{P}\right) \leq\left(\mathcal{O}, C_{Q}\right)$ if and only if $P$ is conjugate to a subset of $Q$.

Lusztig uses the correspondence between conjugacy classes and parabolic subgroups to study a certain map assigning to each nilpotent orbit an element of $\mathcal{N}_{\mathrm{o}, \overline{\mathrm{c}}}$. We now recall the construction of that map, and we consider what can be said about it with the aid of the partial order. Recall that a special piece is the union of a special orbit and all orbits in its closure that are not contained in the closure of any other special orbit. Let $\mathcal{O}$ be a special orbit, and define $\mathcal{M}(\bar{A}(\mathcal{O}))$ to be the set of $\bar{A}(\mathcal{O})$-conjugacy classes of pairs $(x, \rho)$, where $x \in \bar{A}(\mathcal{O})$ and $\rho$ is an irreducible representation of the centralizer of $x$ in $\bar{A}(\mathcal{O})$. There is a natural imbedding of the set of representations in the two-sided cell of the Weyl group that corresponds to $\mathcal{O}$ into the set $\mathcal{M}(\bar{A}(\mathcal{O}))$.

Lusztig's map associates each orbit in the special piece containing $\mathcal{O}$ to some pair $(\mathcal{O}, C)$, by examining the image of the Springer representation of the given orbit under the above imbedding (see [12] and [15]). In particular, $\mathcal{O}$ itself is sent to $(\mathcal{O}, 1)$. One proposition for exceptional groups that appears in [15] is equivalent to the following tidy statement in terms of the partial order on $\mathcal{N}_{\mathrm{o}, \overline{\mathrm{c}}}$.

Proposition 7.3. Let $\mathcal{O}_{1}, \mathcal{O}_{2}$ be two nilpotent orbits in the same special piece, assigned to $\left(\mathcal{O}, C_{1}\right),\left(\mathcal{O}, C_{2}\right)$, respectively, by Lusztig's map. Then $\mathcal{O}_{1} \subset \overline{\mathcal{O}_{2}}$ if and only if $\left(\mathcal{O}, C_{1}\right) \geq\left(\mathcal{O}, C_{2}\right)$.

This is proved in [15] by case-by-case computation, but an appropriate application of the extended duality map renders this proposition obvious, as follows. Sommers' canonical inverse (see Section 2) is conjectured to coincide with Lusztig's map, under an appropriate identification $\bar{A}(\mathcal{O}) \simeq \bar{A}\left(d_{\mathrm{BV}}(\mathcal{O})\right)$. Sommers has verified this conjecture for the exceptional groups. The identification of conjugacy classes in $\bar{A}(\mathcal{O})$ with those in $\bar{A}\left(d_{\mathrm{BV}}(\mathcal{O})\right)$ is order-preserving, because it respects their Coxeter structures. The canonical inverse map itself is order-reversing (Proposition 2.8), so Lusztig's map is order-reversing as well.

Proposition 7.3 is only stated by Lusztig for the exceptional groups because he did not have the correspondence between conjugacy classes and parabolic subgroups of $\bar{A}(\mathcal{O})$ available for classical groups, but the proposition should be true in general.

7.2. Equivariant $\boldsymbol{K}$-theory of the nilpotent cone. Let $\mathcal{N}_{\mathrm{o}, \mathrm{r}}$ be the set of pairs $\{(\mathcal{O}, \rho)\}$, where $\mathcal{O}$ is a nilpotent orbit in $\mathfrak{g}$, and $\rho$ is an irreducible representation of the isotropy group of $\mathcal{O}$ in $G$. Let $\mathcal{N}_{\mathrm{o}, \mathrm{r}}^{0}$ (resp. $\mathcal{N}_{\mathrm{o}, \overline{\mathrm{r}}}^{0}$ ) be similarly defined, except that we take $\rho$ to be a representation of $A(\mathcal{O})$ (resp. $\bar{A}(\mathcal{O})$ ) instead. There are obvious inclusions $\mathcal{N}_{\mathrm{o}, \overline{\mathrm{r}}}^{0} \hookrightarrow \mathcal{N}_{\mathrm{o}, \mathrm{r}}^{0} \hookrightarrow \mathcal{N}_{\mathrm{o}, \mathrm{r}}$, given by pulling back representations. Proposition 7.2 
yields a natural bijection between $\mathcal{N}_{\mathrm{o}, \overline{\mathrm{c}}}$ and $\mathcal{N}_{\mathrm{o}, \overline{\mathrm{r}}}^{0}$. In this section, we consider those sets to be identified; we freely use the partial order, as well as terms like "special," in reference to elements of $\mathcal{N}_{\mathrm{o}, \overline{\mathrm{r}}}^{0}$.

Lusztig and Vogan have independently conjectured the existence of a bijection between $\mathcal{N}_{\mathrm{o}, \mathrm{r}}$ and the set $\Lambda_{+}$of dominant weights of $G$, that should arise by studying the equivariant $K$-theory of the nilpotent cone. This idea has been investigated by Bezrukavnikov [5], [6], Ostrik [16], and the author [1]. In [1], the bijection is established for $G L(n)$ by an explicit combinatorial algorithm. In [6], the bijection is proved in general by a study of perverse equivariant coherent sheaves on the nilpotent cone.

Now, nilpotent orbits in ${ }^{L} \mathfrak{g}$ are labelled by their weighted Dynkin diagrams, which may be regarded as weights for $G$. (The weighted Dynkin diagram of the orbit is the semisimple element of the Jacobson-Morozov $\mathfrak{s l}(2)$-triple for the orbit.) It has been observed that, given an orbit $\mathcal{O} \in{ }^{L} \mathcal{N}_{\mathrm{o}}$, this bijection often sends its weighted Dynkin diagram to some pair $\left(\mathcal{O}^{\prime}, \rho\right)$, where $\mathcal{O}^{\prime}=d_{\mathrm{BV}}(\mathcal{O})$ and, moreover, $\rho$ is a representation that descends to the group $A(\mathcal{O})$. This is mentioned in [8]; a more thorough discussion can be found in Section 3 of [3]. In that paper, a specific conjecture about $\rho$ was made.

We shall now review the conjecture of [3], and examine how to reformulate it using the new language of duality for $\mathcal{N}_{\mathrm{o}, \overline{\mathrm{c}}}$ and $\mathcal{N}_{\mathrm{o}, \overline{\mathrm{r}}}^{0}$. Starting with an orbit $\mathcal{O}$ in a classical group, let $\left(\mathcal{O}^{\prime}, C\right)$ be Sommers' canonical inverse for it. To $C$ one associates a subgroup $H_{C} \subset \bar{A}\left(\mathcal{O}^{\prime}\right)$, by first giving a specific presentation of $\bar{A}\left(\mathcal{O}^{\prime}\right)$ as a Coxeter group, then expressing an element of $C$ as a product of certain simple reflections, and finally taking $H_{C}$ to be the subgroup generated by those simple reflections. Conjecture 3.1 of [3] says that the Dynkin diagram of $\mathcal{O}$ is associated by Lusztig's bijection to a pair $\left(\mathcal{O}^{\prime}, \rho\right)$, where $\rho$ is a representation occurring in $\operatorname{Ind}_{H_{C}}^{\bar{A}(\mathcal{O})} 1$.

As remarked in Section 7.1, the presentation of $\bar{A}\left(\mathcal{O}^{\prime}\right)$ chosen in [3] is the same as that produced by Theorem 7.1. Let us identify $C$ as some $C_{P}$, following Proposition 7.2. Now, $\rho$ in turn is equal to $\rho_{Q}$ for some subset $Q \subset S$. This representation occurs in $\operatorname{Ind}_{H_{C}}^{\bar{A}(\mathcal{O})} 1$ if and only if the trivial representation occurs in the restriction of $\rho_{Q}$ to $H_{C}$, by Frobenius reciprocity. Moreover, the definition of $\rho_{Q}$ turns out to have the consequence that the trivial representation occurs in its restriction to the subgroup $H_{C}$ if and only if $P \subset Q$. Using Proposition 7.2 again, we obtain the following equivalent statement.

Conjecture 7.4 ([3], Conjecture 3.1). The Dynkin diagram of $\mathcal{O}$ is assigned to a pair $\left(\mathcal{O}^{\prime}, \rho\right)$ such that $\mathcal{O}^{\prime}=d_{\mathrm{BV}}(\mathcal{O})$ and $\left(\mathcal{O}^{\prime}, \rho\right) \geq \bar{d}(\mathcal{O}, 1)$.

Sommers ([3], Remark 3.2) has also shown by example that the above inequality can, indeed, fail to be an equality. Namely, if one takes $\mathcal{O}$ to be the subregular orbit in $B_{n}$, then $\mathcal{O}^{\prime}$ is an orbit in $C_{n}$ with $\bar{A}\left(\mathcal{O}^{\prime}\right) \simeq \mathbb{Z} / 2 \mathbb{Z}$. Let $\epsilon$ denote the nontrivial representation of $\bar{A}\left(\mathcal{O}^{\prime}\right)$. It turns out that $\bar{d}(\mathcal{O}, 1)=\left(\mathcal{O}^{\prime}, \epsilon\right)$, but the Dynkin diagram of $\mathcal{O}$ is assigned to $\left(\mathcal{O}^{\prime}, 1\right)$ when $n$ is odd and $\left(\mathcal{O}^{\prime}, \epsilon\right)$ when $n$ is even. Notably, $\left(\mathcal{O}^{\prime}, 1\right)$ is not special for $n \geq 3$. Computed examples suggest that this may be a necessary condition for the inequality above to fail to be an equality. The above conjecture can therefore be refined as follows.

Conjecture $7.4^{\prime}$. The Dynkin diagram of $\mathcal{O}$ is assigned to a pair $\left(\mathcal{O}^{\prime}, \rho\right)$ such that $\mathcal{O}^{\prime}=d_{\mathrm{BV}}(\mathcal{O})$ and $\left(\mathcal{O}^{\prime}, \rho\right) \geq \bar{d}(\mathcal{O}, 1)$, with equality if all pairs $\left(\mathcal{O}^{\prime}, \rho^{\prime}\right)>\bar{d}(\mathcal{O}, 1)$ are special.

7.3. The Springer correspondence. Once we have a partial-order structure for certain local systems on nilpotent orbits, an intriguing avenue of inquiry is the relationship of this structure to representations of the Weyl group, via the Springer correspondence. Of course, the Springer correspondence relates Weyl group representations to elements of $\mathcal{N}_{\mathrm{o}, \mathrm{r}}^{0}$, but we only have a partial order structure on $\mathcal{N}_{\mathrm{o}, \overline{\mathrm{r}}}^{0}$. Nevertheless, we shall put aside this stumbling block for the moment.

The statements below will actually be made in the context of the generalized Springer correspondence, which we now review. Let $W$ be the Weyl group, let $u \in \mathcal{O}$ be a nilpotent element, and let $\mathcal{B}_{u}$ be the variety of Borel subalgebras containing $u$. The original Springer correspondence was obtained by defining an action of $W$ on the top-dimensional cohomology of $\mathcal{B}_{u}$. It turns out that the map $\operatorname{Irr}(W) \rightarrow \mathcal{N}_{\mathrm{o}, \mathrm{r}}^{0}$ is injective but not, in general, surjective. Lusztig [13] extended the correspondence to account for the missing elements of $\mathcal{N}_{\mathrm{o}, \mathrm{r}}^{0}$. In this generalized version, the missing elements correspond to irreducible representations of certain groups $W_{L}^{G}=N_{G}(L) / L$, where $L$ is a "cuspidal" Levi subgroup and $N_{G}(L)$ is its normalizer. Let

$$
\nu: \coprod \operatorname{Irr}\left(W_{L}^{G}\right) \stackrel{\sim}{\rightarrow} \mathcal{N}_{\mathrm{o}, \mathrm{r}}^{0}
$$

be the bijection obtained in this way. 
We now introduce a certain class of subgroups for Weyl groups that will be required for the subsequent discussion. Let $S$ be a set of simple reflections generating $W$. Furthermore, let $s_{0}$ be the reflection corresponding to the highest root in the root system for $W$, and let $S_{0}=S \cup\left\{s_{0}\right\}$. Now, a subgroup generated by a subset of $S$ is called a parabolic subgroup. Let us call a subgroup generated by a proper subset of $S_{0}$ a pseudoparabolic subgroup.

(This is by analogy with Sommers' term pseudo-Levi for a connected reductive subgroup of $G$ corresponding to the root system generated by a given proper subset of $S_{0}$. This seems to be a synonym for endoscopic subgroup, although that term is unappealing when one is not doing any endoscopy theory. The idea for this class of subgroups has, at any rate, been in use for much longer than Sommers' terminology: Spaltenstein [20], for instance, employs them without giving them any name whatsoever.)

The following desideratum for the relationship between representations of the $W_{L}^{G}$ and the partial order was originally suggested by Aubert.

Desideratum 7.5. Let $L$ be a cuspidal Levi subgroup of $G$, let $W=W_{L}^{G}$, and let $W^{\prime}$ be any pseudoparabolic subgroup of $W$. For any irreducible representation $\rho$ of $W^{\prime}$, there is a unique irreducible representation $\pi$ occurring in $\operatorname{Ind}_{W^{\prime}}^{W} \rho$ such that $\nu(\pi) \geq \nu\left(\pi^{\prime}\right)$ for all irreducible representations $\pi^{\prime}$ occurring in $\operatorname{Ind}_{W^{\prime}}^{W} \rho$. Moreover, $\pi$ occurs with multiplicity 1 , and it coincides with the truncated induction $j_{W^{\prime}}^{W} \rho$.

One application of this statement will be the strengthening of known results on the unipotent supports of character sheaves: this is the principal topic of investigation in [2]. In the absence of such a statement, previous treatments of this topic have often relied on assumptions about dimension. For example, in Section 4 of [10], Geck defines a certain class of special representations of pseudoparabolic subgroups, for which it is assumed that $j(\rho)$ is attached to an orbit of larger dimension than any other term of Ind $\rho$, and then establishes a number of results under the assumption that one is only dealing with special representations from this class. Similarly, Lusztig, in Theorem 10.7 of [14], proves a statement asserting the existence of a unique unipotent class of maximal dimension having certain properties. Both of these developments rely on the Springer correspondence, so it seems likely that revisiting them with the help of the above desideratum would lead to a considerable sharpening of the results obtained. In particular, Geck gives an example ([10], Example 6.4) showing what can go wrong with representations not belonging to his class. Desideratum 7.5 ought to allow a rephrasing of his results that would accomodate such examples.

All this discussion is, of course, moot if we do not actually have a partial order on $\mathcal{N}_{\mathrm{o}, \mathrm{r}}^{0}$ : this is the stumbling block that we put aside earlier. In [2], it will be shown how to construct a map $\mathcal{N}_{\mathrm{o}, \mathrm{r}}^{0} \rightarrow \mathcal{N}_{\mathrm{o}, \overline{\mathrm{r}}}^{0}$ that lets one pull back the partial order. Of course, in some respects, the partial order on $\mathcal{N}_{\mathrm{o}, \mathrm{r}}^{0}$ cannot be as nice as that on $\mathcal{N}_{\mathrm{o}, \overline{\mathrm{r}}}^{0}$ : for instance, the sign and trivial representations of a given $A(\mathcal{O})$ might fail to be comparable in $\mathcal{N}_{\mathrm{o}, \mathrm{r}}^{0}$, whereas for $\bar{A}(\mathcal{O})$, the sign representation is always smaller than the trivial one (see Proposition 7.2). This partial order will, however, turn out to satisfy the above desideratum, with corresponding implications for the study of character sheaves.

\section{REFERENCES}

[1] P. Achar, Equivariant coherent sheaves on the nilpotent cone for complex reductive Lie groups, Ph.D. thesis, Massachusetts Institute of Technology, 2001.

[2] P. Achar and A.-M. Aubert, Supports unipotents de faisceaux caractères, in preparation.

[3] P. Achar and E. Sommers, Local systems on nilpotent orbits and weighted Dynkin diagrams, Represent. Theory 6 (2002), 190-201.

[4] D. Alvis, Induce/restrict matrices for exceptional Weyl groups, http://www.iusb.edu/ dalvis/.

[5] R. Bezrukavnikov, On tensor categories attached to cells in affine Weyl groups, arXiv:math.RT/001008.

[6] _ Quasi-exceptional sets and equivariant coherent sheaves on the nilpotent cone, arXiv:math.RT/0102039.

[7] R. Carter, Finite Groups of Lie Type: Conjugacy Classes and Complex Characters, John Wiley \& Sons, New York, 1985.

[8] T. Chmutova and V. Ostrik, Calculating distinguished involutions in the affine Weyl groups, arXiv:math.RT/0106011.

[9] D. H. Collingwood and W. M. McGovern, Nilpotent Orbits in Semisimple Lie Algebras, Van Nostrand Reinhold Mathematics Series, Van Nostrand Reinhold Co., New York, 1993.

[10] M. Geck, Character sheaves and generalized Gelfand-Graev characters, Proc. London. Math. Soc. (3) 78 (1999), $139-166$.

[11] G. Lusztig, A class of irreducible representations of a Weyl group, Nederl. Akad. Wetensch. Indag. Math. 41 (1979), 219-226.

[12] _ Characters of a Reductive Group over a Finite Field, Princeton University Press, Princeton, NJ, 1984.

[13] _ Intersection cohomology complexes on a reductive group, Invent. Math. 75 (1984), 205-272.

[14] _ A unipotent support for irreducible representations, Adv. Math. 94 (1992), 139-179. 
[15] - Notes on unipotent classes, Asian J. Math. 1 (1997), 194-207.

[16] V. Ostrik, On the equivariant K-theory of the nilpotent cone, Represent. Theory 4 (2000), 296-305.

[17] E. Sommers, A generalization of the Bala-Carter theorem for nilpotent orbits, Internat. Math. Res. Notices (1998), no. 11, $539-562$.

[18] _ Lusztig's canonical quotient and generalized duality, J. Algebra 243 (2001), no. 2, 790-812.

[19] , personal communication, 2002.

[20] N. Spaltenstein, Classes Unipotentes et Sous-groupes de Borel, Lecture Notes in Mathematics, no. 946, Springer-Verlag, 1982.

Department of Mathematics, University of Chicago, Chicago, IL 60637

E-mail address: pramod@math.uchicago.edu 